Marcia J. King

Applied Physics Division

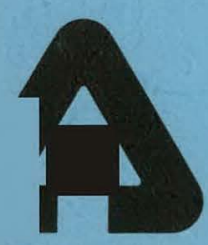




\section{DISCLAIMER}

This report was prepared as an account of work sponsored by an agency of the United States Government. Neither the United States Government nor any agency Thereof, nor any of their employees, makes any warranty, express or implied, or assumes any legal liability or responsibility for the accuracy, completeness, or usefulness of any information, apparatus, product, or process disclosed, or represents that its use would not infringe privately owned rights. Reference herein to any specific commercial product, process, or service by trade name, trademark, manufacturer, or otherwise does not necessarily constitute or imply its endorsement, recommendation, or favoring by the United States Government or any agency thereof. The views and opinions of authors expressed herein do not necessarily state or reflect those of the United States Government or any agency thereof. 


\section{DISCLAIMER}

Portions of this document may be illegible in electronic image products. Images are produced from the best available original document. 
The facilities of Argonne National Laboratory are owned by the United States Government. Under the terms of a contract (W-31-109-Eng-38) between the U. S. Energy Research and Development Administration, Argonne Universities Association and The University of Chicago, the University employs the staff and operates the Laboratory in accordance with policies and programs formulated, approved and reviewed by the Association.

\section{MEMBERS OF ARGONNE UNIVERSITIES ASSOCIATION}

The University of Arizona

Carnegie-Mellon University

Case Western Reserve University

The University of Chicago

University of Cincinnati

Illinois Institute of Technology

University of Illinois

Indiana University

Iowa State University

The University of Iowa
Kansas State University The University of Kansas Loyola University

Marquette University Michigan State University The University of Michigan University of Minnesota University of Missouri Northwestern University University of Notre Dame
The Ohio State University

Ohio University

The Pennsylvania State University

Purdue University

Saint Louis University

Southern Illinois University

The University of Texas at Austin

Washington University

Wayne State University

The University of Wisconsin

\section{NOTICE}

This report was prepared as an account of work sponsored by the United States Government. Neither the United States nor the United States Energy Research and Development Administration, nor any of their employees, nor any of their contractors, subcontractors, or their employees, makes any warranty, express or implied, or assumes any legal liability or responsibility for the accuracy, completeness or usefulness of any information, apparatus, product or process disclosed, or represents that its use would not infringe privately-owned rights. Mention of commercial products, their manufacturers, or their suppliers in this publication does not imply or connote approval or disapproval of the product by Argonne National Laboratory or the U. S. Energy Research and Development Administration. 


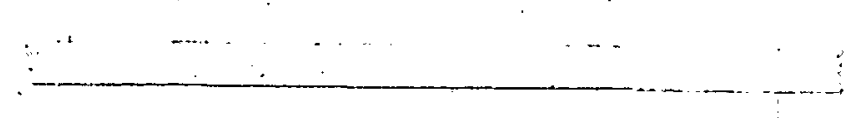

Distribution Category:

LMFBR Fuels and Materials Engineering and Development (UC-79b)

\section{Argonne National Laboratory \\ 9700 South Cass Avenue \\ Argonne, Illinois 60439}

RESPONSE MATRIX METHOD FOR LARGE LMFBR ANALYSIS

by

Marcia J. King

Applied Physics Division

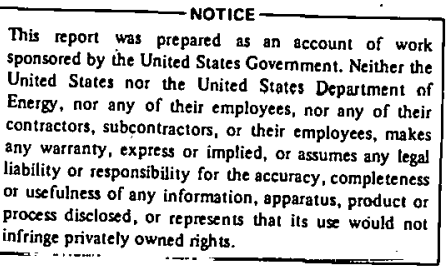

sponsored by the Uncpared as an account of work

United States nor the United States Departient

contractors, sus of their employees, nor any of their

any warranty, express or implied, or assumes, makes

of useful ressonsibility for the aceuracy, completeness

process disclosed, or represents, apparatus, product or

infringe privately owned rights.

June 1977 


\title{
RESPONSE MATRIX METHOD FOR LARGE LMFBR ANALYSIS
}

by

\author{
Marcia J. King \\ Applied Physics Division \\ Argonne National Laboratory \\ Argonne, Illinois 60439
}

\begin{abstract}
The feasibility of using response matrix techniques for computational models of large LMFBRs is examined. Since finite-difference methods based on diffusion theory have generally found a place in fast-reactor codes, a brief rcview of their general matrix foundation is given first in order to contrast it to the general strategy of response matrix methods. Then, in order to present the general method of response matrix technique, two illustrative examples are given. Matrix algorithms arising in the application to large LMFBRs are discussed, and the potential of the response matrix method is explored for a variety of computational problems. Principal properties of the matrices involved are derived with a view to application of numerical methods of solution. The Jacobi tterative method as applied to the current-balance eigenvalue problem is discussed.
\end{abstract}


I. Introduction ........................ 1

II. Approximation to Diffusion Equation by Finite-Difference Techniques - A Qualitative Review ............. 4

III. Response Matrix Method . . . . . . . . . . . . . 7

A. Response-Matrix Equation in One-Dimension . . . . 8

B. Two-Dimensional Hexagonal Bare Reactor With

Triangular rells . . . . . . . . . . . . 12

(1) Response Coefficients for Triangular Cell

in Multigroup Problem . . . . . . . . . 13

(2) Current Balance Equation or Reactor CurrentEIgenvalue Calculation ............ 16

(3) Example of an Iterative Procedure for Calculating the Current Eigenvalue $\lambda$. . . . . 19

IV. Response Matr1x Methods for Large LMFBRs . . . . . . . . 22

A. Hexagonal Ce1.1s ... . . . . . . . . . . . 23

B. A Larger Cell Model . . . . . . . . . . . . . . $2 y$

C. Calculation of Flux and Yower Levels . . . . . . . . 31

V. Summary and Discussions . . . . . . . . . . . . 31

References ..................... . 34

Appendix A. General Properties of the Current-Balance and
Response Matrices . . . . . . . . . 36

Appendix B. A Jacobi Iterative Method for Solving the
Cuxieul Balance Elgenvalue Problem . . . . . . 43

Appendix C. Inversion of the Response Matrix $B_{11} \cdot . \cdot . . . .47$ 


\section{LIST OF TABLES}

No.

I. Example of a Two-Group Current-Balance Solution by the Code CURB for the Two-Dimensional Bare Reactor of Fig. 3. . . . . . . . . . . . . . . . . 49

II. Comparison of Region Volumes for the Reactor Decomposition Shown in Fig. 6. 


\section{LIST OF FIGURES}

No. $\underline{\text { Title }}$

Page

1. Mesh Structure in Two-Dimensional Triangular Geometry

Used In DIF2D . . . . . . . . . . . . . . . 51

2. One-Dimensional Slab Decomposed into Uniform Cells for Application of Response Matrix Method . . . . . . . . . 52

3. One-Sixth of Hexagonal Bare Reactor; Response Matrices are Computed for the Triangular Cells and Used in the Current-Balance Equation to Determine the Interface

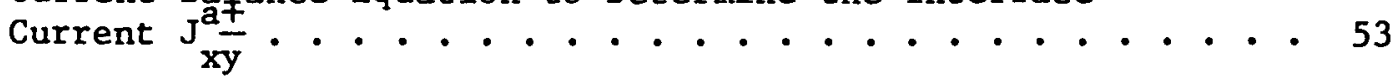

4. Core Layout and Assembly Identification in Hexagonal Geometry . . . . . . . . . . . . . . . . . . 54

5. A Triangular Cell Decomposition of Large Hexagonal Model Reactor . . . . . . . . . . . . . . . . 55

6. Example of Triangular Cell Placement of Control Poison In Model With Triangular Geometry . . . . . . . . . . 56

7. Schematic Diagram for Enrichment Search in Finite-

Difference Method, Two Dimensions . . . . . . . . . .

9. A Portion of the D1rected Graph $G(M)$ for a Two-Dimensional Model Based on Irlañgular Cells . . . . . . . . . . . .

10. A Portion of the Directed Graph G(M) for a Two-Dimensional Model Based on Square Cells . . . . . . . . . . . 60

11. A Purtion of the Directed Graph G(M) for a Two-Dimensional Model Based on Hexagonal Cells 


\section{INTRODUCTION}

For many design constderations in large LMFBRs, such as power shaping by control rod movement, control rod worths, sodium vold reactivities, and heterogeneous cores, spatial dependence plays an important role. In such cases, simplified models such as cylindrical and two-dimensional planar models fail, 1 and it is important to go to an approprlate three-dimensional model; for example, one exhibiting hexagonal-z geometry. It may also be that diffusion theory, so useful for calculation of many global characteristics of the large LMFBR; is not an accurate tool when we are considering local effects which result from the heterogenelty of the core. However, three-dimenstonal models based on, for example, transport theory, are formidable in cost and running time as are even three-dimensional fintte-difference diffusion codes. Thus it becomes Important to consider alternative computational methods which can be applied to three-dimensional models of large LMFBRs.

In this preliminary report, the feasibility of using response matrix techniques for computational models of large LMFBRs is examined. In part, the report is intended to be tutorial in nature for readers who are not famillar with response matrix methods. The discussion has been restricted to calculations based on diffusion theory in order to make a comparison to finite-diffusion codes such as DIF2D. ${ }^{2}$ It is worth noting, however, that one of the advantages of the response matrix method is that diffusion theory and transport theory in approprlate regions of the reactor can be carried out simultaneously. 
In addition to the restriction above, the discussion has been limited to two-dimensional hexagonal models for simpliclty. The method 1s easily generalized to three dimensions and the salient features are obvious from the two-dimensional treatment.

The manner of application of the response matrix technique to be discussed here 18 by no means unique. There have been several veroiono of the method $^{3^{-9}}$ (almost all of which have been applied to light water reactors). No attempt is made to evaluate the different techniques in regard to performance for LMFBR calculations; rather, one method of application is developed which 1llustrates the general steps of the calculation as they might be applied to the large LMFBR.

Baslcally, the response matrix method involves solving for boundary conditions at all interfaces to obtain a solution for the reactor. That is, the system (reactor) is decomposed into a number of simple subunits. In the first stage of the calculation, the response coefficients, or response matrix, are calculated for each type of subunit. This information allows us to calculate outgoing neutron currents of the subunit, or cell resulting from any input currents to the subunit. In the second stage, the calci1iatinn is performed for the reactor as a whole and involves the interface currents of the cells as unknowns. This is referred to as the reactor calculation or the current-balance calculation. Once the second stage is complete, the power and flux levels throughout the reactor may be calculated.

The response matrix method is particularly advantageous for problems Involving cells or subuntts which remaln unchanged in the problem. Examples 
would be blanket regions during an enrichment search; blanket and core regions, i.e., most of the reactor, during criticality searches by control-rod movement. In cases like these, once the response matrix is computed, it may be stored. Instead of laboriously 1terating finite-difference equations in these regions, one is able to use the precalculated response functions.

We shall attempt to clarify and illustrate these points in this report. The outline is as follows. In chapter II, we briefly review pertinent features of finite-difference approximations to solutions to the diffusion equation. This is included partly in order to compare in some measure the methods of response matrices and finlte-dffferences, and partly because, at least in the short run of proposed applications, the cell calculations for large LMFBRs will be based on finlte-difference approximations.

In chapter III, the response matrix method is applied to two examples. The physics of the problem is illustrated by application to a one-dimensional slab; the calculational aspects are fllustrated by consideration of a twodimensional bare reactor, hexagonal in shape with triangular cells. A simple example of an iterative solution to the reactor calculation is given. This chapter is primarily tutorial in nature and can be easily skipped by readers already familiar with response matrix techniques.

A discussion of possible means of application to large hexagonalshaped LMFBRs is contalned in chapter IV. Hexagonal-shaped assembly-sized cells and larger triangular cells are constdered. 
Chapter V includes a summary and discussion and recomendations for response matrix calculations as applied to large LMFBRs.

In Appendix A, the principal properties of the matrices involved in the response matrix method are derived with a view to application of numerical methods of solution, which are discussed in Appendices $B$ and $C$.

II. APPROXIMATIUN 'IU DIFFUSION EQ̇UATION RY FINITH-DIFFERENCE TECHNIQUES - A QUALITATIVE REVIEW

An approximate solution to the multi-group diffusion equation is obtained by imposing a mesh structure on the reactor. The differential equations representing neutron diffusion for each energy group are considered for the Ith cell and integrated over the cell volume (cross sections are taken as constant within the cell). The resulting finite-differenced equations takes the general form 10

$$
\begin{gathered}
{\left[\sum_{R_{1}}^{g}+\sum_{j}^{J} \frac{D_{i j}^{g}}{\Delta_{i j}^{2}}\right] \phi_{1 g}-\sum_{j}^{J} \frac{D_{i j}^{g}}{\Delta_{i j}^{2}} \phi_{j g}-\sum_{g^{\prime}=1}^{g-1} \sum_{S_{i}}^{g^{\prime}+g} \phi_{i g^{\prime}}} \\
=\frac{x^{g}}{k} \sum_{g^{\prime}=1}^{G} v_{g^{\prime}} \sum_{t_{j}}^{g^{\prime}} \phi_{i g^{\prime},}
\end{gathered}
$$

where $\phi_{1 g}$ is the neutron flux in energy group $g$ at the ith mesh point, $\Delta_{1 j}$ is the distance between 1 th and $f$ th mesh point, and the macroscopic 
cross sections $D_{i j}^{g}, \sum_{R_{i}}^{g}, \sum_{s_{i}}^{g^{\prime} \rightarrow g}, x^{g}, \nu_{g}^{\prime} \sum_{f_{i}}^{g^{\prime}}$ are taken as constant within a given region. The sums over the index $j$ are taken over mesh points adjacent to the ith mesh point.

Eq. (2.1) is a set of GXN simultaneous linear algebraic equations, where $\mathrm{N}$ is the number of spatial mesh points and $\mathrm{G}$ the number of energy groups. In matrix form, let us express (2.1) as

$$
\mathrm{M} \phi=\frac{1}{\mathrm{k}} \mathrm{F} \phi \equiv \frac{1}{\mathrm{k}} \mathrm{S} \text {. }
$$

The matrix structure of $M$ depends on the spatial geometry of the reactor model. For example, for one energy group, in $1-D, 2-D$, and $3-D$ geometries, Eq. (2.1) becomes a 3-point, 5-point, and 7-point difference equation, respectively. Thus, for example, in the two-dimenstonal case, the matrix structure of (2.1) would be an NXN block matrix with five diagonals:

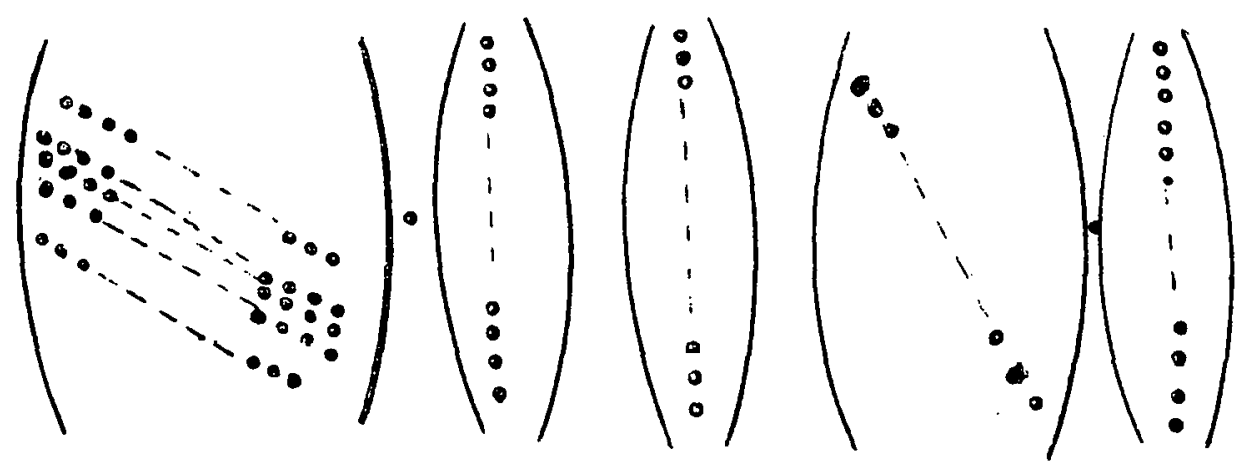

In general, if the flux is normalized at one energy-group space mesh point, there are GXN equations avallable to detcrmine the GXN-1 fluxes and eigenvalue $k$ eff

The elgenvalue problem summarized by Eq. (2.2) can be solved by a number of iterative strategies. It is not our purpose to examine these various techniques but to obtain on overview of the general algorithms 
in order to make a qualitative comparison to response matrix techniques. We brlefly outline the general methodology:10

(1) Take an Initial guess for the source vector $\mathrm{s}^{(0)}$ and eigenvalue $\mathrm{k}^{(0)}$.

(2) Solve the inhomogeneous matrix equation

$M \phi^{(n+1)}=\frac{1}{k}(n) S^{(n)}$

for $\phi^{(n+1)}$, Thts involves the inversion of the large matrix $M$ and must be done by iterative techniques such as the Jacobi-Richardson or Causs-Seidel methods. ${ }^{11}$ These iterations are often denoted as "Inner" iterations.

(3) After the new flux estimate $\phi^{(n+1)}$ is obtained, the next multiplication elgenvalue is estimated through a weighting scheme.

(4) The source tteration is tested for convergence by satisfying an Imposed condition; for example,

$$
\left|\frac{k^{(n+1)}-k^{(n)}}{k^{(n+1)}}\right|<\varepsilon_{1} \text { and }\left|\frac{s^{(n)}-s^{(n-1)}}{s^{(n)}}\right|<\varepsilon_{2}
$$

(5) The fission source $S^{(\mathfrak{n})}$ for the next iteration is chosen by an extrapolation sçheme such as

$$
S^{(n)}=\frac{1}{k}(n) F_{\phi}^{(n)}+\alpha\left[\frac{1}{k}(n) F \phi^{(n)}-s^{(n-1)}\right] .
$$

Fur problems Involving a large number of mesh points, for example, two- and three-dimensional models of large LMFBRs, the matrices are extremely 
unwieldly. There are various methods of dealing with these matrices, such as the channel-ordered method used in the code DIF2D. ${ }^{2}$ In DIF2D, the mesh structure in two-dimensional models is denoted by the pair of indices $(i, j)$ such as shown in Fig. 1. The finite-difference equations are first ordered with respect to channels running from $i=1$ to IMAX. Within each of these channels, they are then ordered with respect to $g=1$ to $G$. Finally, within each energy group, they are ordered with respect to $f=1, \ldots$, JMAX. The resulting eigenvalue equation involves a IXI block matrix tridiagonal in form. The submatrices, which are in turn block matrices, are GXG dense matrices. Each component of the submatrix is in turn a JXJ tridiagonal matrix. In this manner, the problem is broken down into simpler subunits.

\section{RESPONSE MATRIX METHOD}

The response matrix method is best decribed by demonstrating its application to a specific example. As was pointed out in chapter 1 , the cell calculation for the response coefficients can be carried out using any of several methods: e.g., transport theory, Monte Carlo methods, collision probabilities, or diffusion theory. Since we anticipate applying the response matrix method to problems involving large LMFBRs for which previous finite-difference diffusion theory methods have been used, we provide two examples based on diffusion theory. The first, the determination of the criticality condition in a one-dimensional slab, can be solved analytically and is chosen in order to illustrate that the solution is independent of the cell size, i.e., diffusion theory can be applied to calculate response coefficients even if the cell is of the order of the 
neutron free path. This seemingly obvious fact apparently does not have an analogy when transport methods are applied in the cell calculation. 9 The second example is based on a two-dimensional bare hexagonal reactor of uniform composition. Here the currents along the faces of each cell are not constant as they are in the one-dimensional slab, and the response matrix method represents an approximation to the solution. We have chosen the simplest assumption, namely that the interface currents can be approximated by average currents. In practice this approximation can be improved by many methods, e.g. dividing the cell interfaces into subunits over which the currents are taken constant, 10 or assuming a simple functional dependence for the interface currents. $3,10,11$

\section{A. Response-Matrix Equation in One-Dimension}

Cousider a une-dimenstonal slab of unitorm composition and width $A$ as shown in Fig, 2, and assume nnly one neutron velocity. In this case the diffusion equation is a simple boundary value problem which can be solved analytically. Provided the partial currents entering the slab from outside are set to zero to satisfy boundary conditions, wo obtain the criticality condition

$$
\tan \mathrm{BA}=\mathrm{DB} /\left[(\mathrm{DB})^{2}-1 / 4\right]
$$

where $B^{2}=\left(\nu \Sigma_{f}-\Sigma_{a}\right) / D$. We shall retrieve this condition by application of the response matrix equation. 
The slab is divided into I cells, and for simplicity, the same width is assigned to each cell (see Fig. 2). Denote by $\mathrm{J}_{i}^{+}$the partial currents In the $\pm x$ direction at point $x_{1}$, that is

$$
J \frac{ \pm}{1} \equiv 1 / 4 \phi\left(X_{i}\right) \mp \frac{D}{2} \frac{d \phi\left(X_{i}\right)}{d x} \text {. }
$$

Reflection and transmission coefficients $\mathrm{R}_{i}^{+}$and $\mathrm{T}_{i}^{+}$are defined by

$$
\begin{aligned}
& \mathrm{R}_{1}^{+} \mathrm{J}_{i}^{+} \equiv \mathrm{J}_{1, \mathrm{r}}^{-}=\text {contribution to } \mathrm{J}_{i}^{-} \text {due to } \mathrm{J}_{1}^{+} \text {at } \mathrm{x}_{i}, \\
& \mathrm{~T}_{1}^{+} \mathrm{J}_{i}^{+}=\mathrm{J}_{i+1, t}^{+}=\text {contribution to } \mathrm{J}_{i+1}^{+} \text {due to } \mathrm{J}_{1}^{+} \text {at } \mathrm{x}_{i} \cdot
\end{aligned}
$$

Similarly, define $\mathrm{R}_{i}^{-}$and $\mathrm{T}_{i}^{-}$by

$$
\begin{aligned}
& \mathrm{R}_{i}^{-} \mathrm{J}_{i}^{-} \equiv J_{i, r^{\prime}}^{+} \\
& \mathrm{T}_{i}^{-} J_{i}^{-} \equiv J_{i-1, t}^{-}
\end{aligned}
$$

If, for example, $J_{i}^{+}$is a unit current input in the $+x$ direction at point $x_{i}$, then $\mathrm{T}_{i}^{+}$is the resulting contribution to the partfal current in the $+\mathrm{x}$ direction at point $x_{i+1}$.

Provided no external source is present in the region $x_{i+1}-x_{i}$, the following relations hold:

$$
\begin{aligned}
& J_{i+1}^{+}=J_{i+1, t}^{+}+J_{i+1, r}^{+}=T_{i}^{+} J_{i}^{+}+R_{i+1}^{-} J_{i+1}^{-} \\
& J_{i}^{-}=J_{i, t}^{-}+J_{i, r}^{-}=T_{i+1}^{-} J_{i+1}^{-}+R_{i}^{+} J_{i}^{+}
\end{aligned}
$$


In matrix form, let us express these two equations as

$$
J_{1}=R_{1} J_{1+1}
$$

where $\mathrm{J}_{\ell} \equiv\left[\begin{array}{c}\mathrm{J}_{\ell}^{+} \\ \mathrm{J}_{\ell}^{-}\end{array}\right]$,

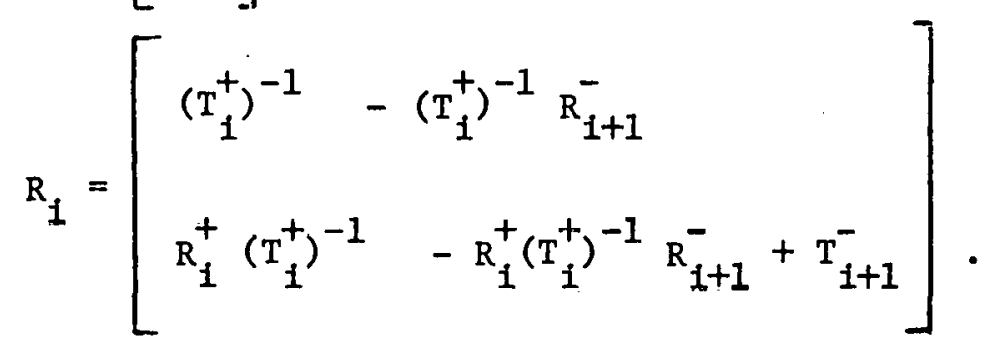

$R_{1}$ is referred to as the response matrix for node(i). For multigroup models, the partial currents $J \frac{t}{1}$ become column vectors with $G$ elements and $\mathrm{R}_{1}^{+}, \mathrm{T} \frac{+}{i}$ are $\mathrm{GXG}$ matrices.

Iterating Eq. (3-8), we obtain

$$
J_{1-1}=k_{i-1} J_{1}=k_{i-1} k_{1} J_{i+1} \text {. }
$$

and, finally,

$$
\mathrm{J}_{1}=\mathrm{R} \mathrm{J}_{\mathrm{I}^{\prime}}
$$

where

$$
R=R_{1} \cdot R_{2} \cdots R_{I}
$$

The boundary conditions at the edges of the slab require $J_{1}^{+}=J_{I}^{-}=0$, or, for a nontrivial solution

$$
\text { (R) }{ }_{11}=0 \text {. }
$$

Eq. (3-14) is the criticality condition.

At this point, we see that for our simple reactor, the problem is reduced to computing the reflection and transmission coeffictents for one 
cell. In order to compare the result (3-14) to (3-1), we apply diffusion theory to calculate these coefficients.

The procedure is as follows. The cell with boundaries at $x_{i}$ and $x_{i+1}$ is treated as if it were isolated in a vacuum, and a unit source of neutrons is imposed on one side, at $x_{i}$. Then,

$$
\begin{aligned}
& \mathrm{J}_{i+1}^{+}=\mathrm{T}_{i}^{+} \mathrm{J}_{i}^{+}=\mathrm{T}_{i}^{+}, \\
& \mathrm{J}_{i}^{-}=\mathrm{R}_{i}^{+} \mathrm{J}_{i}^{+}=\mathrm{R}_{i}^{+} .
\end{aligned}
$$

From symmetry arguments, $T_{i+1}^{-}=T_{i}^{+}, R_{i+1}^{-},=R_{i}^{+}$. Thus, the response matrix for node 1 becomes

$$
R_{1}=\left[\begin{array}{cc}
\frac{1}{J_{i+1}^{+}} & \frac{-J_{i}^{-}}{J_{i+1}^{+}} \\
\frac{J_{i}^{-}}{J_{i+1}^{+}} & -\frac{\left(J_{i}^{-}\right)^{2}}{J_{i+1}^{+}}+J_{i+1}^{+}
\end{array}\right] \text {. }
$$

Applying the boundary conditions

$$
\begin{aligned}
& J_{i+1}^{-}=J^{-}\left(x_{i+1}\right)=0, \\
& J_{1}^{+}=\lim _{x \rightarrow X_{1}} J^{+}(x)=1 / 2,
\end{aligned}
$$

to the solution to the diffusion equation

$$
\frac{\mathrm{d}^{2}}{\mathrm{~d} \mathrm{x}^{2}} \phi(\mathrm{x})+\mathrm{B}^{2} \phi(\mathrm{x})=0,
$$

we finally obtain 


$$
R_{i}=\frac{1}{D B}\left[\begin{array}{lc}
D \cos B a-\left(D^{2} B^{2}-1 / 4\right) \sin B a & -\left(D^{2} B^{2}+1 / 4\right) \sin B a \\
\left(D^{2} B^{2}+1 / 4\right) \sin B a & \frac{D^{2} B^{2}-\left(D^{2} B^{2}+1 / 4\right)^{2} \sin ^{2} B a}{D B \cos B a-\left(D^{2} B^{2}-1 / 4\right) \sin B a}
\end{array}\right],
$$

where $a$ is the cell width. Using $A=I a$ and a simple mathematical proof by induction, we obtain

$$
\begin{aligned}
& R=R_{1} \cdot R_{2} \cdots R_{I} \\
& =\left[\begin{array}{ll}
\cos B A-\frac{\left(D^{2} B^{2}-1 / 4\right)}{D B} \sin B A & \frac{\left(D^{2} B^{2}+1 / 4\right)}{D B} \sin B A \\
\frac{\left(D^{2} B^{2}+1 / 4\right) \sin B A}{D B} & \frac{1-\left(D^{2} B^{2}+1 / 4\right)^{2} \sin { }^{2} B / D^{2} B^{2}}{\cos B A-\left(D^{2} B^{2}-1 / 4\right) \sin B A / D B}
\end{array}\right],
\end{aligned}
$$

and setting $(R)_{11}=0$ ylelds the criticality condition (3.1). Note that the solution is independent of the cell width $a$.

\section{B. Two-Dimensional Hexagonal Bare Reactor With Triangular Cells}

As an example of the extension of the response matrix techniques to two dimensions and to multigroup diffusion problems, a simple hexagonal bare reactor with a single composition has been chosen. This example will also serve as a simple prototype for illustrating methods of calculation sultable for computer codes. The two-dimensional model is exhibited in Fig. 3 where one-sixth of the core layout is displayed. The reactor is divided into triangular cells as shown. 
1. Response Coefficients for Triangular Cell in Multigroup Problem.

(a) Definttion of response coefficients

For the simple bare reactor of Fig. 3, there is only one cell type. The faces of the triangular cell are denoted by the parameter a, where a takes on the value of 1 for the face along the $x$-axis, and $a=2$ and 3 for the subsequent faces taken counterclockvise. Vacuum boundary conditions are applied to two of the cell faces. On the remaining face, vacuum boundary conditions are applied for all energy groups except one mode $g$, for which we impose a unit input current to the cell. The resulting outgoing currents at each face, corresponding to energy groups $g^{\prime}=1, \ldots, G$ are calculated by means of a diffusion code such as DIF2D.

The incoming and outgoing currents for mode $g$ are denoted by $\mathrm{J}_{\mathrm{g}}^{\mathrm{a}-}$ and $\mathrm{J}_{\mathrm{g}}^{\mathrm{at}}$, respectively, where $\mathrm{a}=1,2$, or 3 . We specify one incoming current, say,

$$
\mathrm{J}_{\mathrm{g}}^{\mathrm{a}-} \quad \text { (input) }
$$

for a particular value of $a$ and $g$, and calculate the resulting values of

$$
\mathrm{J}_{\mathrm{g}^{\prime}}^{\mathrm{a}^{\prime}+} \mathrm{g}^{\prime}=1,2, \ldots, \mathrm{G} ; \mathrm{a}^{\prime}=1,2,3 \text {. }
$$

In the actual calculation, $\mathrm{J}_{\mathrm{g}^{\prime}}^{\mathrm{a}^{\prime}+}$ is the average outgoing current along the given face. Repeating the calculation for cell values of $g$ (symetry arguments make it unnecessary to repeat the calculation for more than one side of the cell face), we are then able to define the response coeffictent by

$$
{ }_{g^{\prime} g}^{a^{\prime} a} \equiv J_{g^{\prime}}^{a^{\prime}+} / J_{g}^{a-}
$$


Ordering first with respect to group, we introduce the column vectors whth $G$ components

$$
\mathrm{J}_{-}^{\mathrm{at}}=\left[\begin{array}{c}
\mathrm{J}_{1}^{\mathrm{a}} \\
\mathrm{J}_{2}^{\mathrm{a}} \\
\cdot \\
\mathrm{J}_{\mathrm{G}}^{\mathrm{a}}
\end{array}\right] \text {, }
$$

and the GXG matrices

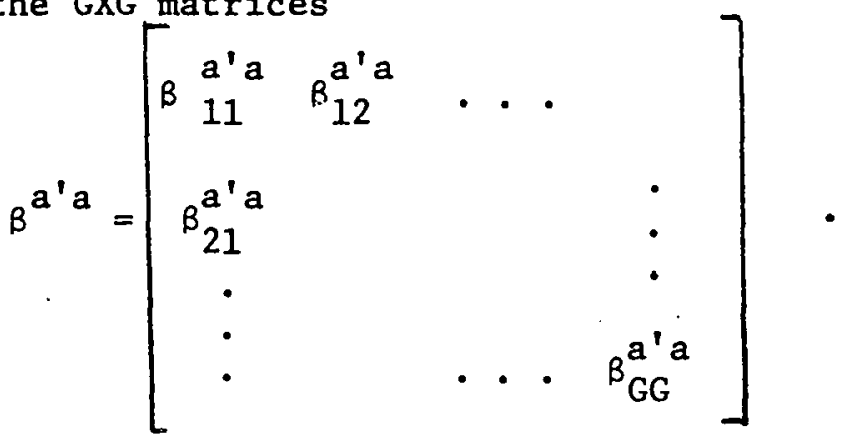

Then forming the 3 -component vectors

$$
\mathrm{J}=\left[\begin{array}{l}
\mathrm{J}^{1 \pm} \\
\mathrm{J}^{2 \pm} \\
\mathrm{J}^{3 \pm}
\end{array}\right]
$$

and the block matrix

$$
B=\left[\begin{array}{lll}
\beta^{11} & \beta^{12} & \beta^{13} \\
\beta^{21} & \beta^{22} & \beta^{23} \\
\beta^{31} & \beta^{32} & \beta^{33}
\end{array}\right] \text {, }
$$


we rewrite (3.24) in the matrix form

$$
\mathrm{J}^{+}=\mathrm{B} \mathrm{J}^{-} \text {. }
$$

The symmetry of the equilateral triangle reduces the number of independent response coefficients for a given $g$ and $g^{\prime}$ to two. We shall define these to be

$$
\beta_{g^{\prime} g}^{r}=J_{g^{\prime}}^{a+} / J_{g}^{a-}
$$

(note $\beta_{g^{\prime} g}^{r}$ will be independent of $a$ ), and

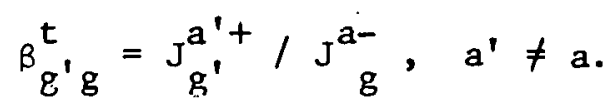

(b) Diffusion calculation for the cell

The multigroup diffusion equation to be solved is

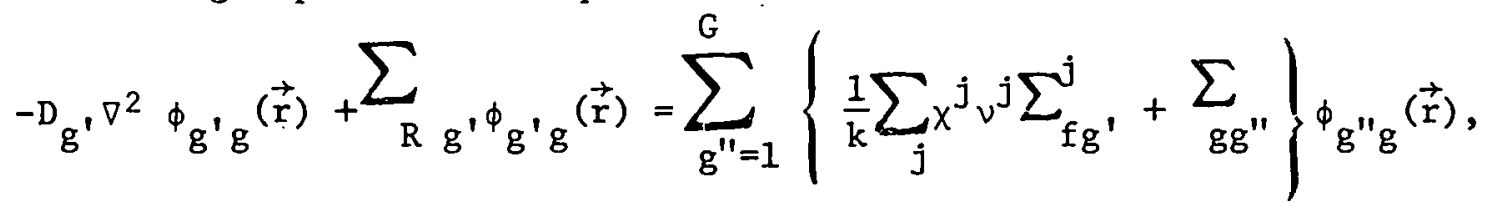

$$
\begin{aligned}
& g, g^{\prime}=1,2, \ldots, G \text {, }
\end{aligned}
$$

where $\phi_{g^{\prime} g}(\vec{r})$ is the flux distribution of neutrons of group $g^{\prime}$ produced by the Incident current of group $g$ through face 1 , say. The parameter $k$, which is the effective multiplication eigenvalue of the reactor, is to be specified in the input to the calculational code. Equation (3.32) is solved subject to the boundary cunditions below:

\section{Face 1}

$$
1 / 4 \phi_{g^{\prime} g}(\vec{r})+\frac{D_{g^{\prime}}}{2} \vec{\varepsilon}_{1} \cdot \nabla \phi_{g^{\prime}}(\vec{r})=1, \vec{r} \text { on face } 1 ;
$$


Face 2

$$
1 / 4 \phi_{g^{\prime} g}(\vec{r})+\frac{D_{g^{\prime}}}{2} \vec{\varepsilon}_{2} \cdot \nabla \phi_{g^{\prime} g}(\vec{r})=0, \vec{r} \text { on face } 2 ;
$$

Form 3

$$
1 / 4 \phi_{g^{\prime} g}(\vec{r})+\vec{e}_{3} \nabla \phi_{g^{\prime} g}(\vec{r})=0, \vec{r} \text { on face } 3 .
$$

$\vec{\varepsilon}_{1}$ is the unit outward normal to face $i$.

Once the solutions for the flux and its partial derivatives are obtained, the average outgoing partial currents are calculated for each face, thus yielding the response coefficients $\beta_{g^{\prime}}^{r} g^{\prime} \beta_{g^{\prime}}^{t} g^{*}$

These calculations may be carried out in a straightforward way for the trlangular cell (as well as other 2-D geometries), for example, by the code DIF2D. ${ }^{2}$ The number of cell mesh points to be used in this fintte-difference calculation will depend on the size of the cells and accuracy desired (althnugh a greatcr number of mesh points does not necessarily ensure greater accuracy of final results, of course, since the currents are averaged over a cell face).

2. Current Balance Equation or Reactor Current-Eigenvalue Calculation For calculational purposes, it is convenfent to formulate the reactor calculation of interface currents as an elgenvalue problem. There appears in the literature a large varlety of methods for such a formulation; here we follow one similar to that used by Pryor and Graves. ${ }^{4}$

The triangular cells are denoted by the xy indices as shown in Fig. 3 (one-sixth - core symmetry is exploited). Currents at the boundary of 
the reactor are not employed, i.e. boundary conditions imply the incoming currents are zero, and the outgoing currents do not appear in the current balance equation.

Ordering first with regard to the energy groups $g, g=1,2, \ldots, G$, we define, in analogy to (3.25),

$$
\mathrm{J}_{\mathrm{xy}}^{\mathrm{a} \pm}=\left[\begin{array}{c}
\mathrm{J}_{\mathrm{xy}}^{\mathrm{a}}, 1 \\
\mathrm{~J}^{\mathrm{a} \pm} \\
\mathrm{xy}, 2 \\
\vdots \\
\mathrm{J}^{\mathrm{a} \pm} \\
\mathrm{xy}, \mathrm{G}
\end{array}\right] \text {, }
$$

and then form the 3-component vectors

$$
J \frac{ \pm}{x y}=\left[\begin{array}{c}
J^{1 \pm} \\
J^{2 \pm} \\
x y \\
J^{3 \pm} \\
x y
\end{array}\right]
$$

For our simple reactor then, we have the response equations

$$
J_{x y}^{+}=B(k) J_{x y}^{-} .
$$

(For a reactor of more than one composition, the response matrix $B$ would depend on the material composition of the $x y$ the cel1). Note that we have expressed the response matrix as an implicit function of the reactor elgenvalue $k$.

Writing out the system of equations (3.38) in full, we would find we have 10 outgoing partial currents $\mathrm{J}_{x y}^{a+}$ in terms of 10 incoming currents 
$\mathrm{J}_{\mathrm{xy}}^{\mathrm{a}-}$. Symmetry reduces the number of independent currents to 4 outgoing and 4 incoming partial currents, which are related as follows:

$$
\begin{aligned}
& \mathrm{J}_{11}^{2+}=\left[2 \beta_{t}^{2} \cdot\left(1-\beta_{\mathrm{r}}-\beta_{t}\right)^{-1}+\beta_{\mathrm{r}}\right] \mathrm{J}_{11}^{2-} \\
& \mathrm{J}_{12}^{1+}=\left(\beta_{\mathrm{r}}+\beta_{\mathrm{t}}\right) \mathrm{J}_{12}^{1-}+\beta_{\mathrm{t}} \mathrm{J}_{12}^{2-} \\
& \mathrm{J}_{12}^{2+}=2 \beta_{\mathrm{t}} \mathrm{J}_{12}^{1-}+\beta_{\mathrm{r}} \mathrm{J}_{12}^{2-} \\
& \mathrm{J}_{13}^{1+}=\left[\beta_{\mathrm{r}}+\beta_{t}^{2-} \cdot\left(1-\beta_{\mathrm{r}}\right)^{-1}\right] \mathrm{J}_{13}^{1-}
\end{aligned}
$$

These expressions for the outgoing partial currents will be incorporated Into the current-balance equation which expresses a conservation law, namely, the outgoing partial current of one cell must equal the incoming partial current in the adjacent cell. The current-balance equation will become an elgenvalue problem if we write

$$
\mathrm{J}^{+}=\lambda \mathrm{AJ}^{-}
$$

where

$$
J \pm \equiv\left[\begin{array}{r}
2+ \\
\mathrm{J}_{11}^{2+} \\
\mathrm{J}_{12}^{1+} \\
\mathrm{J}_{12}^{2+} \\
\mathrm{J}_{13}^{1+}
\end{array}\right]
$$

and 
$A \equiv\left[\begin{array}{llll}0 & 0 & 1 & 0 \\ 0 & 0 & 0 & 1 \\ 1 & 0 & 0 & 0 \\ 0 & 1 & 0 & 0\end{array}\right]$

The current eigenvalue $\lambda$ takes on the value of unity for criticality.

Note that the permutation matrix $A$ is unitary, 1.e.

$$
\mathrm{A}^{-1}=\mathrm{A} \text {. }
$$

Expressing (3.39) in matrix form, we write

$$
\mathrm{J}^{+}=\beta \mathrm{J}^{-}
$$

where

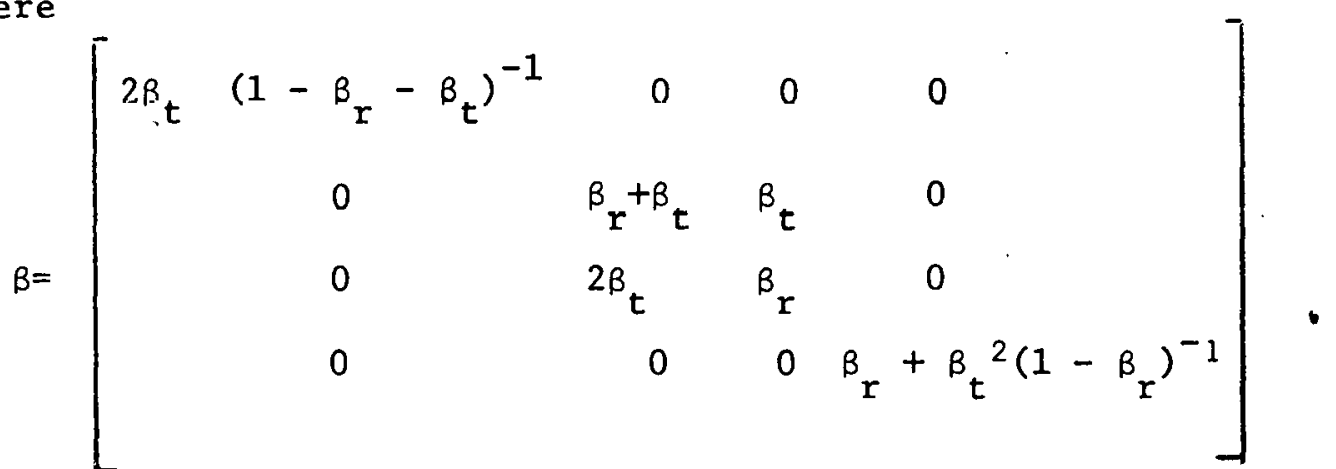

Thus we obtain the eigenvalue problem

$$
A^{-1} B(k) J^{-}=\lambda J^{-} \text {. }
$$

3. Example of an Iterative Procedure for Calculating the Current Eigenvalue $\lambda$ The solution to the current-balance eigenvalue problem evolved in the last section may be obtained through any of several iterative methods. We have chosen here the method of simultaneous displacements as it is applied to eigenvalue problems.12 The elgenvalue equation (3.46) is rewritten as

$$
M(k) J^{-}=\lambda J^{-}
$$


where we have set $M=A^{-1} B$. We first note that $M$ is of the form

$$
M=L+U \text {, }
$$

that is, the diagonal elements of $A$ are zero. For this case, then, the method of simultaneous displacements yields the iteration rule (see Appendix B)

$$
\mathrm{J}^{-(\mathrm{n}+1)}=\frac{1}{\lambda}(\mathrm{L}+\mathrm{U}) \mathrm{J}^{-(n)}
$$

that is,

$$
J^{-(n+1)}=\frac{1}{\lambda} M(k) J^{-(n)} .
$$

The iteration must not only lead to solutions for the elgenvectors but must continually improve the approximation to the elgenvalue $\lambda$. We employ two concurrent iterations - one for the eigenvector and one for the eigenvalue.

Assume initial guesses $\mathrm{J}^{-(0)}$ and $\lambda^{(0)}$. After a sufficient number of of iterations, we obtain

$$
J^{-(n)} \approx \gamma_{1} J^{-(n-1)}
$$

where $\gamma_{1}$ is the eigenvalue of the largest magnitude of the matrix $B(k, \lambda) \equiv \frac{1}{\lambda} M(k)$. Once $\gamma_{1}$ is obtained, the value of $\lambda$ is adjusted: $\lambda$ is increased or decreased according to whether $\gamma_{1}$ is greater or less than unity, respectively. This procedure is continued until $\lambda$ and $\mathrm{J}^{-}$satisfy imposed convergence criteria. For criticality, $\lambda$ must equal unity. First and second guesses of the multiplication factor $k$ can be used to yield an extrapolation scheme to achieve $\lambda=1$. 
As we noted, Eq (3.51) is valid provided

$$
\left|\gamma_{1}\right| \geq\left|\gamma_{1}\right|, \quad 1=2,3, \ldots,
$$

where $\gamma_{1}, 1=2,3, \ldots$, are the remaining eigenvalues of $B(k, \lambda)$. For our example, however, it can be demonstrated that if $\gamma_{1}$ is an eigenvalue, $-\gamma_{1}$ is also one. Thus it is necessary to replace (3.51) by the approximation

$$
J^{-(n)} \approx \gamma_{1}^{2} J^{-(n-2)}
$$

and estimate $\gamma_{1}$ by taking

$$
\gamma_{1} \approx+\left[J^{-(n)} / J^{-(n-2)}\right]^{1 / 2}
$$

The appropriate 1terated current elgenvector which converges to a solution of (3.47) is given by

$$
J_{n}^{-} \equiv 1 / 2\left[J^{-(n)}+J^{-(n-1)}\right] \text {. }
$$

This cyclic behavior, which results in pairs of roots $\pm \gamma_{1}$, is characteristic of response matrix current eigenvalue problems involving triangular or square cells. The cyclic properties of current balance matrices are discussed in Appendix A.

The above iteration scheme provided the basis for a sample code for solving the eigenvalue equation (3.47) for the bare hexagonal reactor of Fig. 3. The code CURB was written for an arbitary number of energy groups. To test the code, an arbitrary twö-group response matrix was selected. The input to the code and results are summarized in Table $I$. The initial guess for $\lambda$ was unity; for the current vectors, all components for the initial 
guess were unity. Six iterations were imposed for the estimate of $\gamma_{1}$ via Eq. (3.54). A decision tree algorithm was chosen to obtain Improved guesses for $\lambda$ for each search pass, with

$$
\lambda \leftarrow \lambda \pm \Delta \lambda \text {. }
$$

$\Delta \lambda$ was 0.5 for the first pass and then was reduced by a factor of one half for each subsequent search pass. For the two cases in the Table, the convergence criteria for $\lambda$ was taken to be $U .0001$ and 0.001 , respectively; convergence was obtained after 14 and 19 search passes, respectively. The resulting current eigenvector and value for $\lambda$ are found in the Table.

\section{RESPONSE MATRIX METHODS FOR LARGE LMFBRS}

A typical core layout for a carblde-fueled LMFBR of the 5000 MWt class is displayed in Fig. 4. Since the neutron meanfree path is large $(\sim 5 \mathrm{~cm}$ for this reactor), many performance evaluatlons of such reactors have been based on the finite-difference diffusion codes DIF1D, 13 DIF2D, ${ }^{2}$ and (in 1iulled amount) DIF3I. 14 For hexagonal geometries, in the horizontal plane, six mesh points per hexagonal assembly are imposed in DIF2D and DIF3D (the diffusion code VENTURE ${ }^{15}$ utilizes one mesh point per assembly in the hexagonal geometry version; $6,24, \ldots$ mooh points pes assembly it triangular geometry is imposed).

In this chapter, we explore the potential of the response matrix method as a calculational tool for such reactors. In section $A$, the hexagonal assembly volumes are taken as subunits or cells for the response coefficient calculations. The structure of the resulting current-balance equations are discussed. In section $B$, the reactor is decomposed into triangular cells 
characterized by volumes one and a half times larger than the assembly volumes. This not only reduces the number of unknowns but simplifies the structure of the current-balance equations. (In the diffusion codes, e.g. DIF2D, the material composition for a given region of the reactor is homogenized and the calculation makes no distinction between assemblies in a given region. Thus we can divide such a region into arbitrarily shaped cells).

In section $C$, calculation of power and flux levels in the reactor is discussed.

\section{A. Hexagonal Cells}

The most suggestive and straight-forward application of the response watrix method is to decompose the model into hexagonal cells corresponding to the actual hexagonal assemblies. In this case, the number of unknown interface partial currents is the same as the number of unknown fluxes used in the code DIF2D, i.e., 6 unknowns per hexagonal cell. Since the interface current is constant over an area roughly equivalent to the mesh point spacing, it is expected that the accuracy of the two methods will be comparable. Using the model reactor of Fig. 4 as a prototype, we examine the steps involved in applying response matrix techniques.

The core is characterized by two radial zones with different plutonium enrichments, and 18 assembly positions designated for control rods. The core is surrounded by a radial blanket and a reflector. Allowing for the presence or absence of control poison, there are six regions differing in composition. Therefore, response coefficients must be calculated for six 
cell types. Each cell is a regular hexagon. Vacuum boundary conditions are applied to all but one face, where a untt input current for mode $g$ is imposed. The response coefficients are calculated and yleld the GXG matrices

$$
B_{z}^{a^{\prime} a} ; a^{\prime}, a=1,2, \ldots 6 ; z=1,2, \ldots, 6 .
$$

The Indices $a^{\prime}$ and a denote the faces for outgoing and incoming currents, respectively, while the index $z$ refers to the cell type, i.e., Inner or outer core, etc. From these matrices, we construct the $6 \times 6$ block matrices $B_{z}$ :

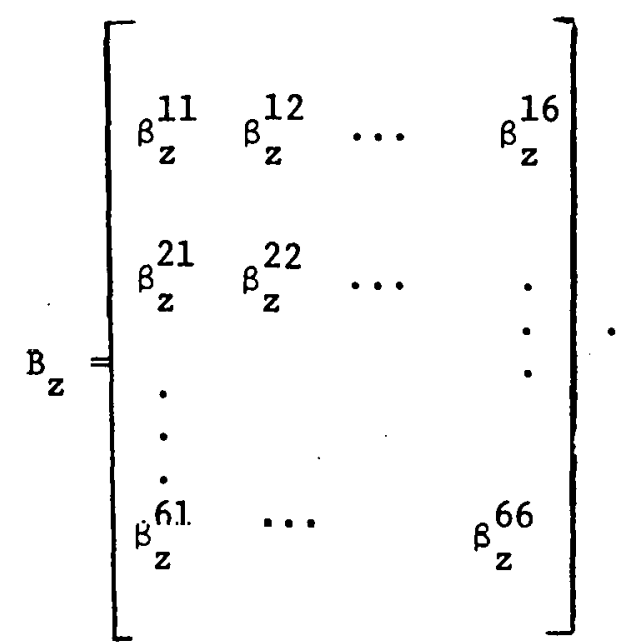

The six matrires $B_{\bar{z}}$, then, are $(6 X G) \times(6 X G)$ dense matrices. However, Just as for the triangular cells of the last chapter, symmetry arguements reduces the number of independent GXG matrices $B_{z}^{a^{\prime} a}$ to 4 for fixed $z$.

The response matrices $B_{z}$ are implicit functions of the reactor eigenvalue $k$. There are two ways of calculating $k$. One is to expand the response matrices in terms of inverse powers of $k$ and include the calculation of $k$ in the reactor calculation. ${ }^{4,6}$ This eliminates the necessity of repeating the cell calculations but at the expense of complicating the cell calculation and the current-balance equations. The second way is to calculate the response 
matrices for more than one value of $k$ and extrapolate to the efoenvalue which ylelds $\lambda=1$. Here we consider the latter.

The reactor calculation, or current-balance elgenvalue problem can be formulated in the manner described in chapter 3 . The response equations are then summarized by

$$
\mathrm{J}^{+}=\mathrm{B}(\mathrm{k}) \mathrm{J}^{-} \text {, }
$$

where $\mathrm{J}^{+}$are $\mathrm{N}$ component vectors

$$
\pm=\left[\begin{array}{c}
J \frac{t}{11} \\
J \frac{t}{12} \\
\cdot \\
j \frac{t}{i j} \\
\cdot \\
\cdot \\
J \frac{ \pm}{I J}
\end{array}\right] \text {, }
$$

where $N$ is the number of assemblies. The $1 \mathrm{fth}$ indices refer to the $1, \mathrm{fth}$ hexagonal cell. Symmetry arguments can be used to reduce the number of components. However, here we treat the problem more generally. The matrix $B(k)$ In (4.3) is a block diagonal matrix:

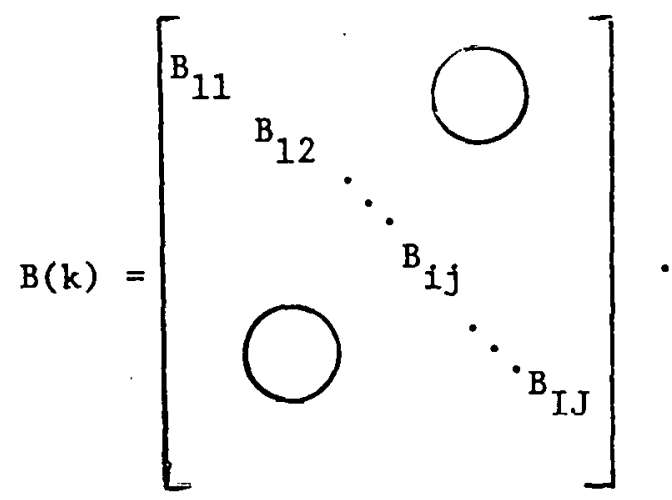


There will be a large number of equalities between the components $B_{i j}$ since there are only six different cell types.

The components $J \frac{+}{1 j}$ are themselves vectors. We write, for the internal cells (see Appendix B for more details and a discussion of boundary cells),

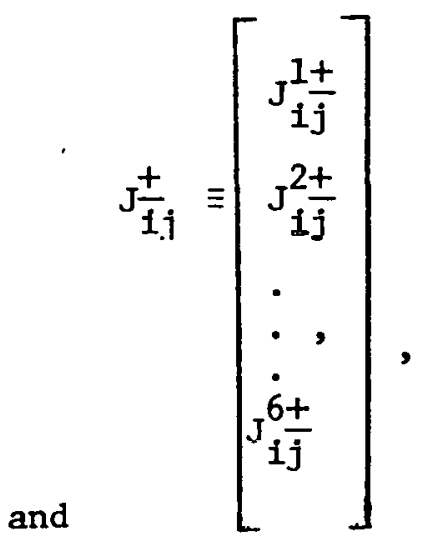

$$
B_{i j}=\left[\begin{array}{cccc}
\beta_{1 j}^{11} & \beta_{i j}^{12} & \cdots & \beta_{i j}^{16} \\
\beta_{1 j}^{21} & \beta_{1 j}^{22} & \cdots & \\
\cdot & \cdot & & \\
\cdot & \cdot & & \\
\beta_{1 j}^{61} & & & \beta_{1 j}^{66}
\end{array}\right] \text {, }
$$

in analogy to the constructions of chapter 3 . The 6 dimensions correspond to the six faces of the cell. The submatrices. $\beta_{i j}^{a a^{\prime}}$ are not all independent. Properties of the matrices $B_{i j}$ which arise from rulational and reflection symmetries are discussed in Appendix A. These properties reduce considerably the computations necessary to invert $B_{i j}$ (see Appendix $C$ ). 
Finally, $J_{i j}^{a t}, a=1, \ldots 6$, are $G$-component vectors and $\beta_{1 j}^{a^{\prime} a}$ are, correspondly, GXG matrices.

The current-balance equation is given by

$$
\mathrm{J}^{+}=\lambda \mathrm{AJ}^{-}
$$

where $A$ is a permutation matrix of the type encounted in chapter III and is unitary, 1.e., $A^{-1} A=1$ (see Appendix A).

Thus we obtain the reactor current-eigenvalue equation:

$A^{-1} B(k) J^{-}=\lambda J^{-}$.

We consider now in more detall the steps involved in solving (4.9) such that the current eigenvalue $\lambda$ takes on the value of unity. The iteration of cell calculation, i.e., whether the calculation of a response matrix can be stored or will have to be repeated, depends on the type of problem involved. Four examples are discussed: (1)calculation of $\mathrm{k}_{\text {eff }}$; (2)plutonium enrichment search; (3) control rod movement in two-dimensional models; and (4) control rod movement in three-dimensional models. In all these cases, the response coefficients for cells composed of reflector composttions and those corresponding to control rod positions fllled with sodium are calculated once and stored.

(a) k-Effective Calculation

Response matrices corresponding to an initial and second guess for $k$ are performed for the three cells corresponding to the two enrichment zones of the core and the radial blanket. Response matrices for the cells with control poison is calculated once and stored. The reactor calculation is carried out for the two values of the elgenvalue $k$. Using the resulting values of $\lambda$, an 
extrapolation scheme to obtain $\lambda=1$ yields a third guess for $k$. The procedure is repeated unt11 an imposed convergence criterla is satisfied.

(b) Plutonium Enrichment Search

Response matrices for control rod and blanket cells are calculated and stored. The ratio of the inner to outer core enrichments is fixed. Response matrices for the two core cells and the current-balance calculations are iterated for different values of the inner core enrichment unt1l the convergence criterion for $\lambda=1$ is satisfled.

(c) Control Rod Movement in Two-Dimensional Models

The multiplication eigenvalue $\mathrm{k}$ is assigned a fixed value, e.g., $\mathrm{k}=1$. Response matrices for all cells except the control position cell are calculated and stored. The response matrlx for the control rod cell and the currentbalance calculation are iterated for different poison concentrations until the convergence criterion for $\lambda=1$ is satisfled.

(d) Control Rod Movement in Three-Dimensional Models

Although we have not considered chree-dimeuslumal mitels explieitly in this report, one of the motivations of the study was the desirability to find alternative computational methods for use in three dimensions. In this context it is worth noting that the response matrix method has one of its greatest potentials for calculations involving control rod movement in and out of the reactur. Assume all other reactor compositions are held fixed; for example, criticality searches at a time node during a burnup calculation. The response matrices for all cell types are calculated once and are stored. Only the reactor calculation is repeated to correspond to 
new boundaries of the control rod region such that $\lambda=1$.

Some comments are due on the use of two elgenvalues to characterize the reactor, namely the multiplication eigenvalue $k$ and the current eigenvalue $\lambda$. The introduction of $k$ into the multigroup diffusion equation (2.1) is a device to ensure that the mathematics leads to a unique physical solution to the equation. It has the effect of replacing each $\nu_{g}$ by $\nu_{g} / k$, so that there always exists a unique positive value of $k$ such that the reactor is "critical". The current elgenvalue $\lambda$ can be interpreted in the same manner if we consider that the response matrix $B(k)$ is replaced by $B(k) / \lambda$ in Eq. (4.9). Thus, there is no reason why we couldn't eliminate the parameter $k$ altogether. 'lhen, of course, many calculations would be conslderably simpler. For example, control rod worths could be calculated in terms of $\Delta \lambda$. However, since the use of the eigenvalue $k$ is well established, we have considered its specification or evaluation as part of the calculational problem.

\section{B. A Larger Cell Model}

In section $A$, the surface subdivision of the prototype reactor was of the same order as the mesh point separation for DIF2D. It has been argued that this usually is not necessary ${ }^{8}$ and that equivalent accuracy can be obtained with larger spatial cells in the response matrix method. We note that if the criterion for accuracy is relaxed, there are other options which can be readily utilized such as synthesis methods, e.g., the code SYN3D.16 Keeping this in mind, we shall consider how models such as the one displayed In Fig. 4 might be broken down into larger cells. 
For the hexagonally-shaped LMFBR, the question is how to decompose the model into larger cells. Examination of Fig. 4 convinces us that we cannot slmply use larger hexagons. Several hexagonal assemblies could be grouped together, and/or cells of different sizes could be assembled. However, such complications as "jagged" boundaries, loss of symmetry of cell shape or composition, cells of varying size, will all result in more involved cell and reactor calculations. It is possible that these disadvantages might be outweighed by having drastically fewer subunits in the modela. More detailed investigation would be necessary to resolve these questions.

Here we consider a rather simple way to model the reactor such that the most pertinent characteristics (hexagon shape, discrete control rods, general power density distributions) are retained, and at the same time a cell structure larger and simpler than the hexagonal cell is imposed. A mathematical model with a hexagonally-shaped boundary is constructed from a serles of triangular cells which are one and a half times larger than the hexagonal assembly cells. An example is displayed in Fig. 5, where this triangular based model is superimposed on the usual hexagonal model. Comparisons of the resulting volumes of various regione of the reactor are compared in Table II and demonstrate the triangular model call be quice realistic. The placement of control rods presents some difficulty, but could be handled, for example, by allowing a triangle to represent a region containing a control rod position as 1llustrated in Fig. 6. Certainly such a model, extended to three dimensions, would give more accurate results for power peaking factors and other spatially dependent parameters than presently used two-dimensional hexagonal or $r-z$ models. 
In addition to the reduction of the number of unknown interface currents, the triangular based model results in substantial simplifications of the response matrix calculations and the current-balance equations. The matrix $B$ appearing in Eq. (4.3), for example, becomes a diagonal block matrix. with a substructure of ( $3 X G) \times(3 X G)$ dense matrices.

\section{Calculation of Flux and Power Levels}

In many applications, it is destrable to know the neutron flux and the power distribution throughout the reactor. Once the currentbalance eigenvalue problem has been solved such that $\lambda=1$, the average flux and power levels at interface boundaries are easily calculated from the interface partial currents. For flux levels within the cell, the diffusion code (or transport code, etc.) can be invoked for each cell with the boundary conditions obtalned from knowledge of the interface currents.

\section{SUMMARY AND DISCUSSION}

The spatial dependence of some reactor parameters has eliminated the use of simple cylindrical or 2-D hexagonal models for many calculations Involving large LMFBRs. 'l'hèse Include power peaking analyses, evaluation of control-rod worths, sodium void reactivities, control-rod movement, and computations for weakly coupled heterogeneous cores. An examination of the method of response matrices has been presented here in order to evaluate its potential as a computational tool for such calculations. Since finite-difference methods based on diffusion theory 2213,14 have generally found a place in fast-reactor codes, we have brlefly reviewed their general. matrix foundation in order to contrast it to the general strategy of 
response matrix methods. The schematic flow diagrams displayed in Figs. 7 and 8 for a plutonfum enrichment search fllustrate the steps involved in the two methods. The main characteristic of the finite-difference approach is the necessity of returning to the beginning of a major step in the calculation or the beginning of the calculation itself for each iteration. The' strategy of the response matrix method in the other hand is to break the large calculation down into smaller blocks in such a way that some, at least, of these blocks can be calculated once and then stored: they are not involved in the iteration scheme. In the example of Fig. 8, these blocks are the diffusion-theory calculation of response matrices for control, blanket, and reflector regions. If the problem at hand is the calculation of the multiplication factor $k_{\text {eff }}$, however, the iteration must return to new inputs for $k$ near the top of the flow diagram. Then all cells are included in the iteration, and if there is an advantage over the comparable finite-difference approximation, it may reside in a faster convergence of the problem. ${ }^{3,9}$ This, however, needs further investigation. It should be kept in mind, also, that $k_{\text {eff }}$ calculations could be eliminated altogether by using the current eigenvalue $\lambda$ as a similar "measure" (see Section IV). The use of larger subunits or cells in the response matrix method would also yield an advantage over finite-difference techniques in calculating $k_{e f f}$, but comparable accuracy must be demonstrated. On the other hand, for three-dimensional calculations where $k_{\text {eff }}$ is fixed, say at $k_{\text {eff }}=1$, and control rods are moved up and down for power shaping and maintaining criticality, provided the vertical cell spacing ylelds proper resolution, none of the cell calculations are involved in 
the iterations: the structure of the diagonal matrix $\mathrm{B}^{-1}$ in Fig. 8 Is modified by assigning the submatrices $\mathrm{B}_{z}^{-1}$ to different positions for each iteration until $\lambda=1$. It appears that the response matrix method in this case offers a distinct advantage over finite-difference techniques. There are, of course, many unresolved questions. One, for example, is the sensitivity of the current eigenvalue $\lambda$ to small changes in the reactor elgenvalue $k$. It is assumed that such problems can be resolved by appropriate modifications to the simple procedure presented in this report. Although the discussion has been limited to diffusion theory applications, we have seen that the decoupling of the cell calculation from the current-balance calculation implies that each cell can be calculated by the appropriate method. For weakly coupled heterogeneous cores, for example, the thick blanket between radial core zones results in large gradients in the radial neutron flux distribution. Thus, a combination of diffusion theory and transport theory cell calculations in the response matrix method could be used to give an accurate description of the neutron diffusion in the reactor. Even for homogeneous cores, the use of transport calculations in regions involving large flux gradients, for example in the radial blanket, should improve accuracy.

In regard to heterogeneous cores, it is worth pointing out that the response matrix method has an additional advantage over finite-difference techniques because of the large number of blanket assemblies. Response matrices for these regions remain unchanged during cnrichment searches for peak to average power optimization.

In conclusion, then, we can say that it appears there are several applications involving large LMFBRs for which the response matrix method 
appears to be a promising calculational tool. It is suggested that a sample problem such as illustrated in Figs. 7 and 8 for a typical 2-D model (see Fig. 4) be examined in more detail, and that the method should be seriously considered as a calculational tool for large heterogeneous reactors.

\section{REFERENCES AND FOOTNOTES}

1. M. J. King "Sensitivity of Power Peaking Analysis to Läige Reactor Core Modcling," ANL-AFP-32, ÜEóber $19 \% 6$.

2. T. A. Daly, G. V. Leaf, and A. S. Kennedy, "The ARC System TwoDimensional Diffusion Theory Capability, DARC2D," ANL-7716, May 1972.

3. K. Aoki and A. Shimizu, J. Nuc. Sc1. and Tech. 2, 149 (1965).

4. R. J. Pryor and W. E. Graves, CONF-730414-P2, p. VII - 179 (1973).

5. H. Sterling Balley, CONF-730414-P2, p VII - 187 (1973).

6. R. J. Pryor, CONF-750413-I, p. 169 (1975).

7. J. M. Sicillan and R. J. Pryor, CONF-750413-I, p. VI - 103 (1975).

8. C. T. McDaniel, CONF-750413-I, p. V-III (1975).

9. An overview of response matrix methods and a more extensive set of references is given by A. Leonard, CoNF-7501113-I, p. III - 15 (1975).

10. See, for example, A. Henry, "Nuclear-Reactor Analysis," MIT Press, Cambridge, Mass (1975), and J. J. Duderstadt and L. J. Hamilton, "Nuclear Reactor Analysis," John Wiley and Sons, Inc., 1976.

11. Z. Weiss and S. 0 . Lindah1, Nuc. Sci. and Eng. 58, p. 166 (1975).

12. M. Clark and K. F. Hansen, "Numerical Methods of Reactor Analysis," Nuclear Science and Technology, 3, Academic Press (1964). 
13. D. E. Neal, G. K. Leaf, and A. S. Kennedy, "The ARC System OneDimensiona1 Diffusion Theory Capability, DARC1D," ANL-7715, May 1971.

14. K. Derstine, "DIF3D, A Multigroup Multidimensional Finite Difference Diffusion Theory Module," ANL Intra-Laboratory Memo, February, 1976.

15. D. R. Vondy, T. B. Fowler, and G. W. Cunningham, "VEnTURE: A Code Block for Solving Multigroup Neutronics Problems Applying the FiniteDifference Diffusion-Theory Approximation to Neutron Transport," ORNL-5602, October 1975.

16. C. H. Adams, "SYN3D - A Single-Channel, Spatial F1ux Synthesis' Code for Diffusion Theory Calculations," ANL-76-21, July (1976).

17. R. S. Varga, "Matrix Iterative Analysis," Prentice-Hall (1962). 
Appendix A. General Propertles of the Current-Balance and Response Matrices

The response equation expressed by (4.3),

$$
\mathrm{J}^{+}=\mathrm{BJ}^{-} \text {, }
$$

and the current-balance equation (4.8) (w1th $\lambda=1$ ),

$$
\mathrm{J}^{+}=\mathrm{AJ}^{-}
$$

are easily generalized to a model comprised of an arbitrary number of cells of arbitrary shape. In this Appendix, we explore anme of the properties of $A, B$, and the matrix $M \equiv A^{-1} B$ for 2-D models involving regular polyhedron decomposition. In practice, these are limited to (1) triangles, (2) squares, and (3)hexagons.

The structure of the current vectors $\mathrm{J}^{+}$can be expressed as follows. Define the $\mathrm{N}$-component vectors

$$
\mathrm{J} \pm=\left[\begin{array}{c}
\mathrm{J} \frac{t}{1} \\
\mathrm{~J} \frac{t}{2} \\
\cdot \\
\mathrm{J} \frac{ \pm}{\mathrm{N}}
\end{array}\right],
$$

where $\mathrm{N}$ is the member of cells in the reactor. If $\mathrm{n}$ is the number of faces on each cell, then for non-boundary, or internal cell, the components $J \frac{t}{i}$ are n-component vecturs:

$$
\mathrm{J} \frac{ \pm}{1}=\left[\begin{array}{c}
1 \pm \\
\mathrm{J}_{1}^{1 \pm} \\
\mathrm{J}_{1}^{2 \pm} \\
\cdot \\
\mathrm{J}_{1}^{\mathrm{n} \pm}
\end{array}\right] \text {. }
$$


Currents on faces which comprise the boundary of the reactor are not Included in Eqs. (A.1) and (A.2), so that the vectors $J \frac{t}{1}$ for boundary cells will have fewer than $n$ components.

Finally, if the number of energy groups is $G$, the components $j_{1}^{a \pm}$ are G-component vectors:

$$
\mathrm{J}_{1}^{\mathrm{a} \pm}=\left[\begin{array}{c}
\mathrm{J}_{1,1}^{\mathrm{a}} \\
\mathrm{J}_{1,2}^{\mathrm{a}} \\
\\
\mathrm{J}_{1, \mathrm{a}}^{\mathrm{a}}
\end{array}\right]
$$

With a similar partitioning, the matrix B is an NXN block.diagonal. matrix of the form

$$
B=\left[\begin{array}{lllll}
{ }^{B} 11 & & & \\
& B_{22} & & & \\
& & & & \\
& & & \\
& & & { }^{B_{N N N}}
\end{array}\right] \text {. }
$$

The submatrices $B_{11}$ for internal cells are nxn dense blocks matrices which we express as

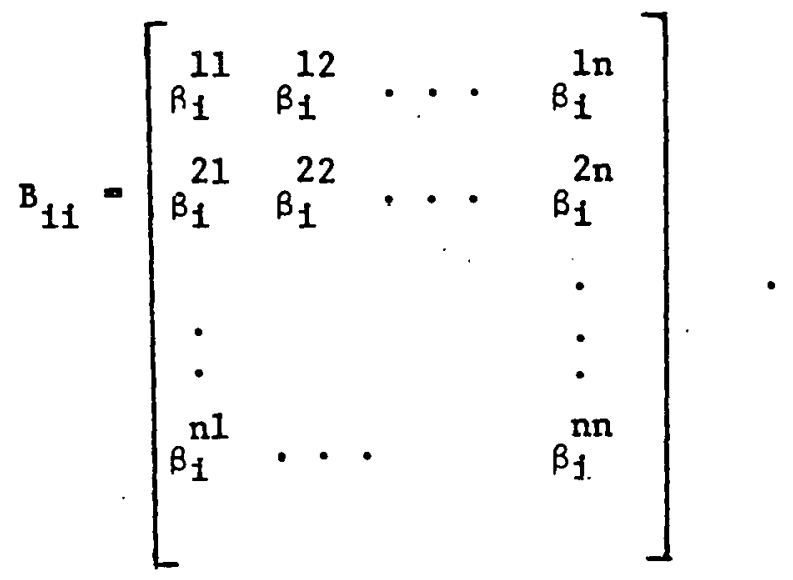


For boundary cells, $B_{i 1}$ are $(n-m) \times(n-m)$ dense blocks, matrices, where $m$ is the number of cell faces lying on the boundary of the reactor. Finally, the entries $B_{1}^{2 a^{\prime}}$ are GXG dense matrices.

Equation (A.2) expresses current conservation across the faces of the cells. A is partitioned permutation matrix partioned in the manner of \pm and B; that 1s, A is a partitioned, or block, matrix which in each row and each colum has some one unit matrix entry, all others zesu.

1) The Unitary and Symmetric Matrix A

The outgoing current from a cell through a given interface, $\mathrm{J}_{i}^{\mathrm{a}}$, must equal the corresponding incoming current in the adjacent cell, $J_{I^{\prime}}^{a^{\prime}-}$. This current conservation is expressed by (A.2). Clearly, from symmetry, 1t follows that

$$
\mathrm{J}^{-}=\mathrm{AJ}^{+}
$$

so that $A^{2}=1$, or $A=A^{-3} 1$. Sinre $\left(J^{-}\right)^{T}\left(J^{-}\right)-\left(J^{+}\right)^{T}\left(J^{+}\right)$, we àlso have $A=A^{T}$.

2) Propertico of the Respunse Matrix B

The components $\beta_{i, g^{\prime} g^{\prime}}^{a^{\prime}}$ defined in analogy to (3.24), are given by

$$
\beta_{i, g^{\prime} g}^{a^{\prime} a}=J_{1, \dot{g}^{\prime}}^{\lambda^{\prime}+} / J_{1, g^{\prime}}^{2-}
$$

and are Llierefore real and non-negative. It follows that the response matrix $B$ is nonsingular, 1.d., $B^{-1}$ exists. The inversion of $B$ is discussed in Appendix $C$.

The use of regular polyhedra to represent the cells in the response matrix method enables us to exploit the symmetries for calculational 
purposes. Invariance under the reflection group $\sigma$ yields the relations

$$
\beta_{1}^{a^{\prime} a}=B_{i}^{a a^{\prime}}
$$

Invariance under the rotation group $C_{n}(n=3,4$, or 6$)$ results in the following form of the matrix $\mathrm{B}_{1}$ :

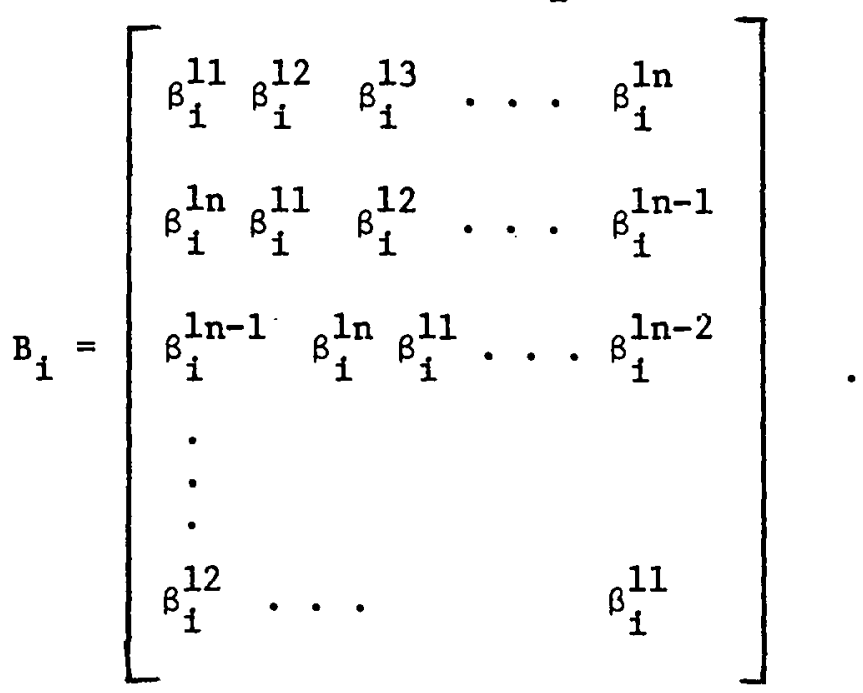

All diagonals are comprised of equal entries. This symmetry in the components of $B_{1}$ reduces the computations involved in inverting $B_{i}$ by $a$ factor of $\mathrm{n}$ if the Jordan or the Gauss elimination method is employed (see Appendix C)

Combining the rcoults of the reflection and rotation symmetries, we obtain the relations

$$
\left.\begin{array}{ccc}
1 n & \\
\beta_{1}^{1 n} & \beta_{1}^{12} \\
\beta_{1}^{1 n-1} & =\beta_{i}^{13} \\
\beta_{1}^{1 n-2} & =\beta_{1}^{14} \\
& \cdot \\
12 & \cdot \\
\beta_{1}^{12} & \beta_{1}^{1 n}
\end{array}\right\} .
$$


3) Irreductbility, Non-Negative and Cyclic Properties of

The Matrix $M=A^{-1} B$

We partition the matrix A to correspond to the partitioning of the vectors \pm and the matrix B. That is, A is partitioned into an NXN block matrix with $n \times n$, or $(n-m) \times(n-m)$, component matrices $A_{i j} \cdot$ The diagonal of $A$ is zero, 1.e., $A_{11}=0$. Therefore, $M$ is also a zero-diagonal block matrix, 1.e.,

$M_{\text {i1 }}=0$.

Further, since $B \geq 0$, it follows that $M \geq 0$, 1.e., the matrix $M$ is non-negative.

It can be demonstrated that $M$ is also irreducible. Its directed graph $G(M)$ has an interesting representation based on the physical relationship of an outgoing current through a particular face of a cell to the incoming currents to the cell; that is, the outgoing current is expressible in terms of the incoming currents to the cel1. Figs. 9, 10, and 11 display portions of the directed graphs $G(M)$ of $M$ for triangular, rectangular, and hexagonal cell compositions, respectively. Points on the graph represent currentes through the adjacent cell fare and also the nodco of $C(M)$. Since every point of the graph can be conncctcd to every otlier pulnt by means of a directed path, $M$ is said to be "strongly connected," and therefore $M$ is irreductble.

The face that $M$ is non-negative and irreducible implies the following important properties: ${ }^{17}$

1) $M$ has a positive real eigenvalue equal to its spectral radius $\rho(\mathrm{M})$. 
2) To $\rho(M)$ there corresponds an eigenvector $x>0$.

3) $\rho(M)$ increases when any entry of $M$ increases.

4) $\rho(M)$ is a simple elgenvalue of $M$.

We will make use of these properties in Appendix B where we Identify the current elgenvalue $\lambda$ with $\rho(M)$.

From a theorem on the bounds of the spectral radius of a non-negative irreducible matrix, 17 we have for the pxp point matrix $M=m_{1 j}$ either

$$
\rho(M)=\sum_{j=1}^{p} m_{i j}
$$

for every $1 \leq 1 \leq p$, or

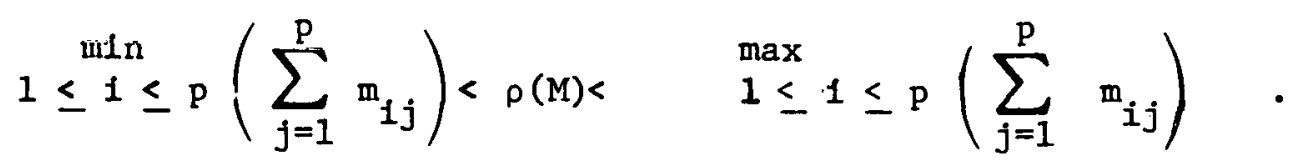

An important property of $M$ follows from a theorem concerned with the cyclic nature of non-negative irreducible matrices: 17

Theorem: Let $M=\left(m_{i j}\right) \geq 0$ be an irreducible pxp matrix, with $G(M)$ as its directed graph. For each node $P_{1}$ of $G(M)$, consider all closed paths connecting $P_{1}$ to itself. If $S_{1}$ is the set of all the lengths $1_{i}$ of these closed paths, let $k_{i}$ be the greatest common divisor of the lengths, i.e.,

$$
\begin{aligned}
& k_{1}=\text { g. c. d. }\left\{1_{1}\right\}, \quad 1 \leq i \leq p . \\
& \quad 1_{1} \in s_{i}
\end{aligned}
$$

Then $k_{1}=k_{2}=\ldots=k_{n}=k$, where $k=1$ when $M$ is primitive, and $k>1$ when $M$ is cyclic of index $k$. 
Examination of the directed graphs in Figs. 9, 10, and 11, leads to the conclusion that for triangular and rectangular cells, $M$ is cyclic of index 2, whereas for hexagonal cells, $M$ is primitive. If the model is extended to three-dimensions by defining cells of rectangular axial cross-sections, M retains the same cyclic properties for the various $x y-p l a n e$ geometries. The consequences of the cyclic properties of $M$ on the iterative methods for solving the current-balance eigenvalue problem are discussed in Appendix B. The most important consequence of a cyclic matrix $M$, where $M \geq 0$ and $M$ is Irreducible, is that there are $k$ elgenvalues of modulus $\rho(M)$ and such that

$$
\rho(M) \exp \left[1\left(\frac{2 \pi j}{k}\right)\right], \quad 0 \leq j \leq k-1
$$

In fact, the characteristic polynomial $\phi(t)=\operatorname{det}(t I-M)$ has the form

$$
\left.\phi(L)=t^{m}\left[e^{k}-\dot{\rho}^{k}(M)\right)\right]\left[t^{k}-\gamma_{2} \rho^{k}(M)\right] \cdot \cdot \cdot\left[t^{k}-\gamma_{r} \rho^{k}(M)\right],
$$

where

$$
\left|\gamma_{1}\right|<1 \text { for } \mid<1 \leq r \text { if } r>1
$$




\section{Appendix B. A Jacobi Iterative Method for Solving the}

\section{Current Balance E1genvalue Problem}

The method of solution to $\mathrm{MJ}^{-}=\lambda \mathrm{J}^{-}$used to solve the prototype problem discussed in Section III was based on the method of simultaneous displacements or Jacobl iterative method. This method as applied to the general current-balance eigenvalue problem is analyzed in more detail below.

As was noted in Appendix $A$, since $M=A^{-1} B$ is a non-negative irreducible matrix,

1. $M$ has a positive real efgenvalue equal to its spectral radius P. (M) ;

2. To $\rho(M)$ there corresponds an elgenvector $x>0$;

3. $\rho(M)$ increases when any entry of $M$ increases;

4. $\rho(M)$ is a simple eigenvalue of $M$.

Because of the above.properties, we will associate the current eigenvalue $\lambda$ with the spectral radius $\rho(M)$. Although we haven't attempted a general proof that $\lambda$ will always be the largest eigenvalue of $M$, it is possible to show that it is in the one-dimensional problem discussed in Section III. Consider the matrix equation

$$
(M-\lambda I) J=0
$$

(we have suppressed the minus index on $J$ for convenience). In the method of simultaneous displacements (or Jacobi iterative method) the Jacobi or Iteration matrix is defined in terms of the decomposition

$$
A=I+D+U,
$$


where $L$ and $U$ are strictly lower and upper matrices, respectively, $D$ is a diagonal matrix, and $A$ is the matrix appearing in $A x=k$, the system of linear equations to be solved. The Jacobi matrix assoclated with $A$ is given by

$$
B=-D^{-1}(L+U)
$$

In our case, we 1dentify $A=M-\lambda I$, so that

$$
B=\frac{1}{\lambda} M \text {. }
$$

The iterative method is given by

$$
\mathrm{J}^{(\mathrm{m}+1)}=B J^{(\mathrm{m})} \text {. }
$$

If all the eigenvalues of $B$ are less than unity, the iteration will aways converge to the trivial solution. If the eigenvalue is greater than unity, the iteration diverges. Therefore it is necessary to continually modify the current-eigenvalue $\lambda$ after each iterative step in order to, convergc to an elgenvalue of unity for $B$. The actual method of application of this iterative procedure depends on the cyclic nature of the matrix $M$.

Suppose we expand the trial solution $\mathrm{J}^{(0)}$ in terms of the eigenvectors of $B$, the complete set heing, say, $j_{r}$. Then, applying (B.j), we obtain

$$
J^{(m)}=\sum_{r}{ }_{r}\left(r_{r}\right)^{m} j_{r},
$$

with $\gamma_{r}$ the efgenvalue of $B$ associated with $j_{r} \cdot$ Eq. (B.6) can be rewritten as

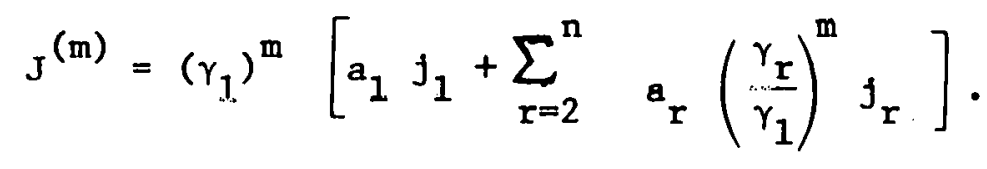


If $\left|\gamma_{1}\right|>\left|\gamma_{2}\right| \geq\left|\gamma_{3}\right| \geq \ldots \geq\left(\gamma_{n}\right)$, then for $m$ large enough and provided $B$ is primitive (i.e., M primitive),

$$
J^{(m)} \simeq \gamma_{1} J^{(m-1)}
$$

If $B$ is cyclic (M cyclic), (B.8) does not hold. If the cyclic index of $B$ is two, we can estimate $\left|\gamma_{1}\right|$ by using

$$
\mathrm{J}^{(\mathrm{m})} \simeq \gamma_{1}^{2} \mathrm{~J}^{(\mathrm{m}-2)}
$$

The convergence rate to $\gamma_{1}$ in both cases above is determined by the factor $\left(\left|\gamma_{2}\right| /\left|\gamma_{1}\right|\right)^{m}$ where $r_{2}$ is the eigenvalue of $B$ with the second largest magnitude. We could accelerate this convergence by using an alternative estimate of $\gamma_{1}$ such as below:

i) Primitive $M$

$$
\frac{\left[J^{(m)}\right]^{T}\left[J^{(m)}\right]}{\left[J^{(m)}\right]^{T}\left[J^{(m-1)}\right]} \simeq \gamma_{1} .
$$

ii) $\mathrm{M} \mathrm{Cyclic}$ of Index 2

$$
\frac{\left[\mathrm{J}^{(\mathrm{m})}\right]^{\mathrm{T}} \mathrm{J}^{(\mathrm{m})}}{\left[\mathrm{J}^{(\mathrm{m})}\right]^{\mathrm{T}} \cdot\left[\mathrm{J}^{(m-2)}\right]} \simeq \mathrm{\gamma}_{1}^{2}
$$

In both cases, the convergence rate depends on the factor $\left(\left|\gamma_{2}\right| /\left|\gamma_{1}\right|\right)^{2 m}$ (this can be determined by substitution of $J^{(0)}=\sum_{r} a_{r} \gamma_{r} f_{r}$ ).

Once the $m$ iterations have ylelded an approximate value for $\left|\gamma_{1}\right|$, the next step is to adjust the value of $\lambda$ to bring $\gamma_{1}$ closer to unity. A decision-tree algorithm was used for the simple prototype problem 
discussed in Section III. $\lambda$ was increased or decreased according to whether $\gamma_{1}$ was greater than or less than unity, respectively. An alternative cholce is to use a weighting scheme to determine the new value of $\lambda$. For M a primitive matrix, we can write

$$
\lambda^{\prime}=\lambda \frac{\sum_{i} J_{1}{ }^{(m)}}{\sum_{i} J_{i}^{(m-1)}},
$$

while for $M$ eyclis of Index 2,

$$
\lambda^{\prime}=\lambda \frac{\sum_{i} J_{1}(m)}{\sum_{1} J_{1}(m-2)} .
$$




\section{Append1x C. Inversion of the Response Matrlx B 11}

In Appendix A, we showed that the matrix $B_{11}$ for a regular polyhedral cell takes the form (for internal cells)

$$
B_{i 1}=\left[\begin{array}{ccccc}
\beta_{i}^{11} & \beta_{i}^{12} & \beta_{i}^{13} & \cdots & \beta_{i}^{1 n} \\
\beta_{1}^{1 n} & \beta_{i}^{11} & \beta_{i}^{12} & \cdots & \beta_{i}^{1 n-1} \\
\beta_{i}^{1 n-1} & \beta_{i}^{1 n} & \beta_{i}^{11} & \cdots & \beta_{i}^{1 n-2} \\
\cdot & & & & \\
\cdot & & & & \vdots \\
\beta_{i}^{12} & \beta_{i}^{13} & \beta_{i}^{14} & \cdots & \beta_{1}^{11}
\end{array}\right] .
$$

If $C_{1 i}$ is the matrix satisfying $B_{1 i} C_{i i}=1$, it is not difficult to show $C_{i i}$ takes the analogous form:

$$
c_{i i}=\left[\begin{array}{ccccc}
c_{i}^{11} & c_{i}^{12} & c_{i}^{13} & \cdots & c_{1}^{1 n} \\
c_{i}^{1 n} & c_{i}^{11} & c_{i}^{12} & \cdots & c_{i}^{1 n-1} \\
\cdot & & & & \cdot \\
c_{i}^{12} & c_{i}^{13} & c_{i}^{14} & \cdots & c_{i}^{11}
\end{array}\right] \cdot
$$

Therefore the elements of $\mathrm{C}_{i 1}$ may be obtained by solving the set of equations

$$
B_{11} x=e,
$$


where $e$ is a column vector with one entry of unity and all other entries are zero, e.g.,

$$
\mathrm{e}=\left(\begin{array}{l}
1 \\
0 \\
0 \\
\dot{0} \\
0
\end{array}\right)
$$

Rotational symmetry reduces the number of equatinns. to be solvod [i.c., system (C.3)] by a factor of $n$, wlieit $n$ is the number of sides to the cell.

$$
\text { Since } B_{11} \text { is a dense matrix, either the Gauss elimination or the }
$$
Jordan method would be approprlate for the solution of (C.3). Once the vector $x$ is obtained, $C_{11}=B_{11}^{-1}$ is eas1ly constructed. 
TABLE I. Example of a Two-Group Current-Balance Solution by the Code CURB for the Two-Dimensional Bare Reactor of F1g. 3. Arbitary Reflector and Transmission

$$
\text { Matrices, } \beta_{r}=\left(\begin{array}{ll}
0.150 & 0.008 \\
0.002 & 0.050
\end{array}\right), \beta_{t}=\left(\begin{array}{ll}
0.550 & 0.015 \\
0.005 & 0.100
\end{array}\right) \text {, were chosen for input. }
$$

\section{INPUT}

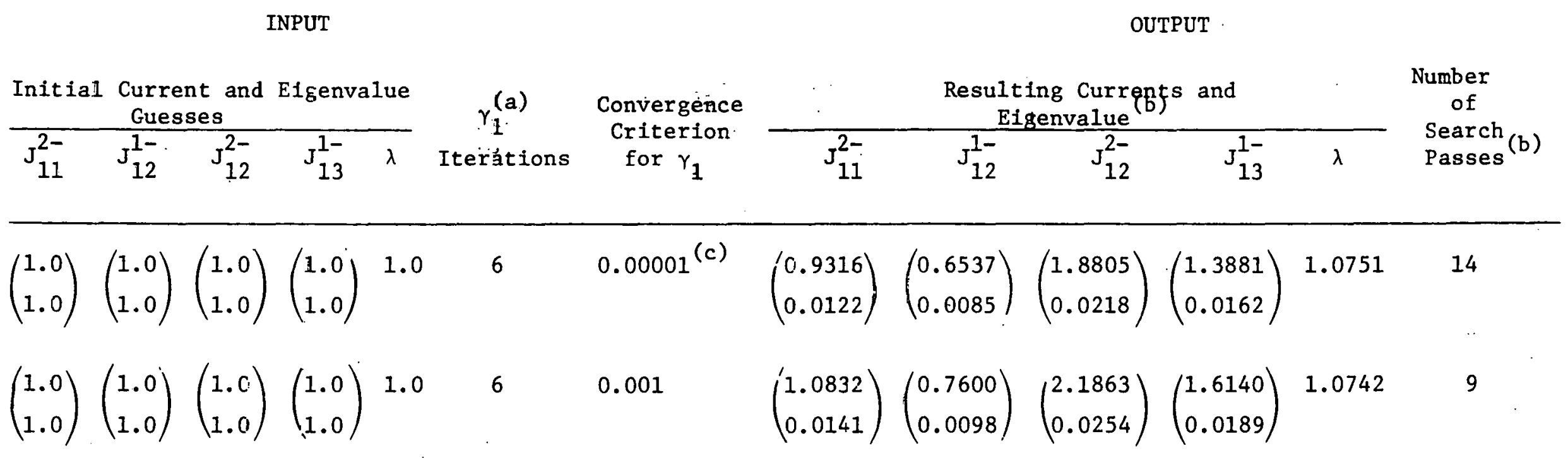

\section{Criterion} for $\gamma_{1}$
OUTPUT

$$
\begin{aligned}
& \left(\begin{array}{l}
0.9316 \\
0.0122
\end{array}\right)\left(\begin{array}{l}
0.6537 \\
0.0085
\end{array}\right)\left(\begin{array}{l}
1.8805 \\
0.0218
\end{array}\right)\left(\begin{array}{l}
1.3881 \\
0.0162
\end{array}\right) 1.0751 \\
& \left(\begin{array}{l}
1.0832 \\
0.0141
\end{array}\right)\left(\begin{array}{l}
0.7600 \\
0.0098
\end{array}\right)\left(\begin{array}{l}
2.1863 \\
0.0254
\end{array}\right)\left(\begin{array}{l}
1.6140 \\
0.0189
\end{array}\right) 1.0742
\end{aligned}
$$

(a) See text for explanation of $\gamma_{1}$ iterations and search passes.

(b) Arbitrary normalization of current eigenvectors.

(c) A convergence criterion of 0.0001 yielded identical results, implying that the decision-tree algorithm ended at the same step for both runs. 
TABLE II. Comparison of Region Volumes for the Reactor Decomposition Shown 1n F1g. 6. Trlangular Cells are 1-1/2 times Larger than Hexagonal Ce11s

\begin{tabular}{lcccc}
\hline & & & Volumes & (Arbitary Normalization) \\
\cline { 2 - 5 } & Inner Core & Outer Core & Total Core & Radial Blanket \\
\hline Hexagonal Geometry & 271 & 198 & 469 & 156 \\
Triangular Geometry & 270 & 198 & 468 & 153
\end{tabular}




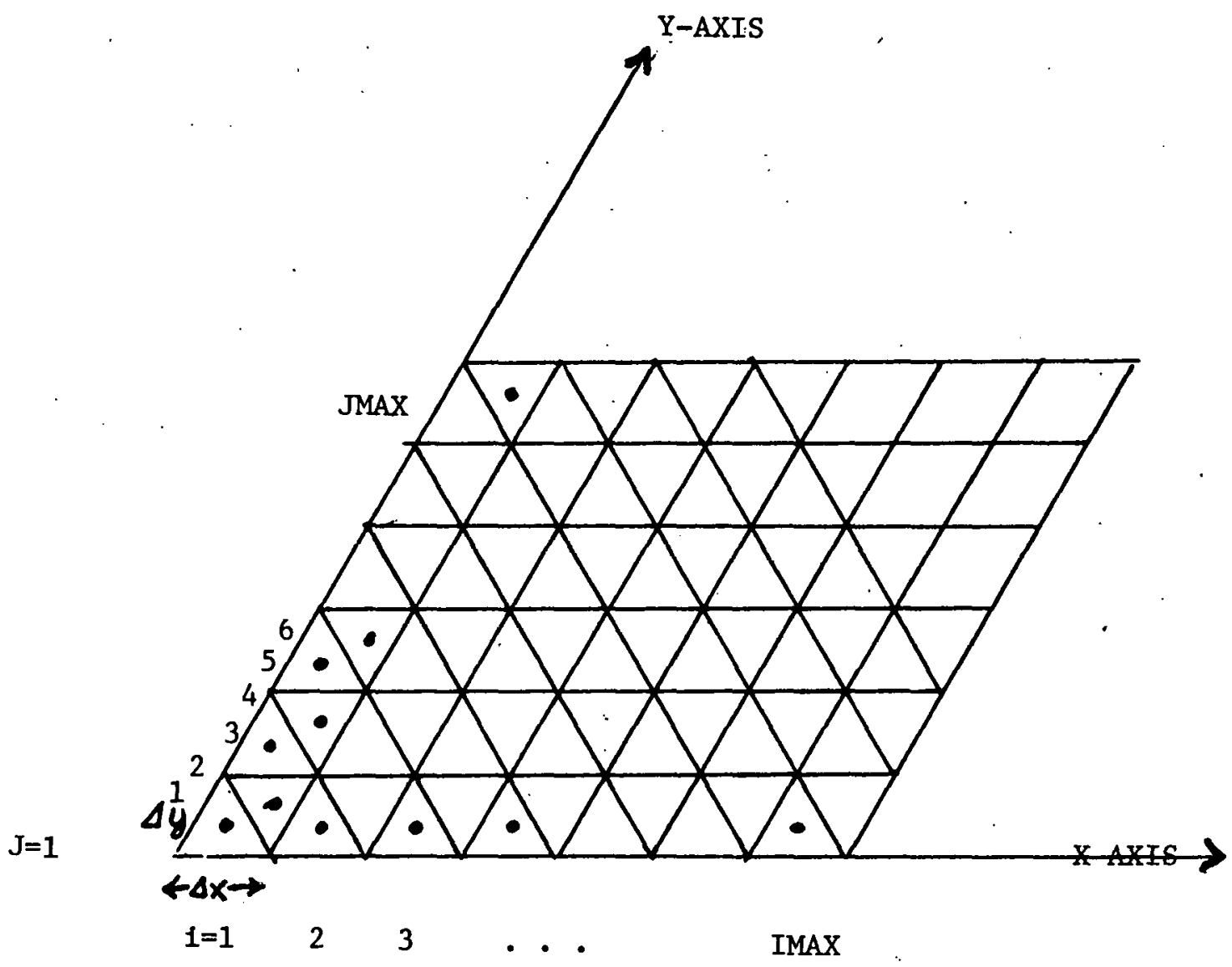

Fig. 1. Mesh structure in two-dimensional triangular geometry used in DIF2D. 


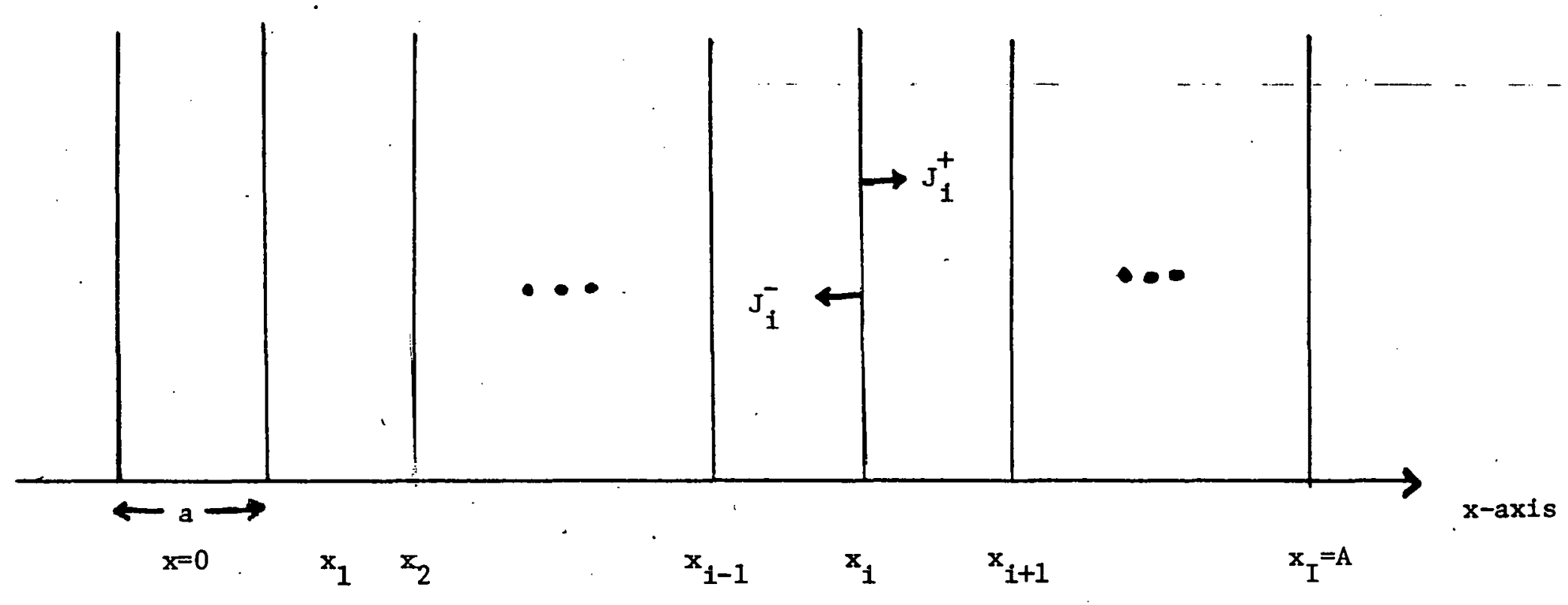

Fig. 2. One-dimensional slab deccmposed into uniform cells for application of response matrix method. 


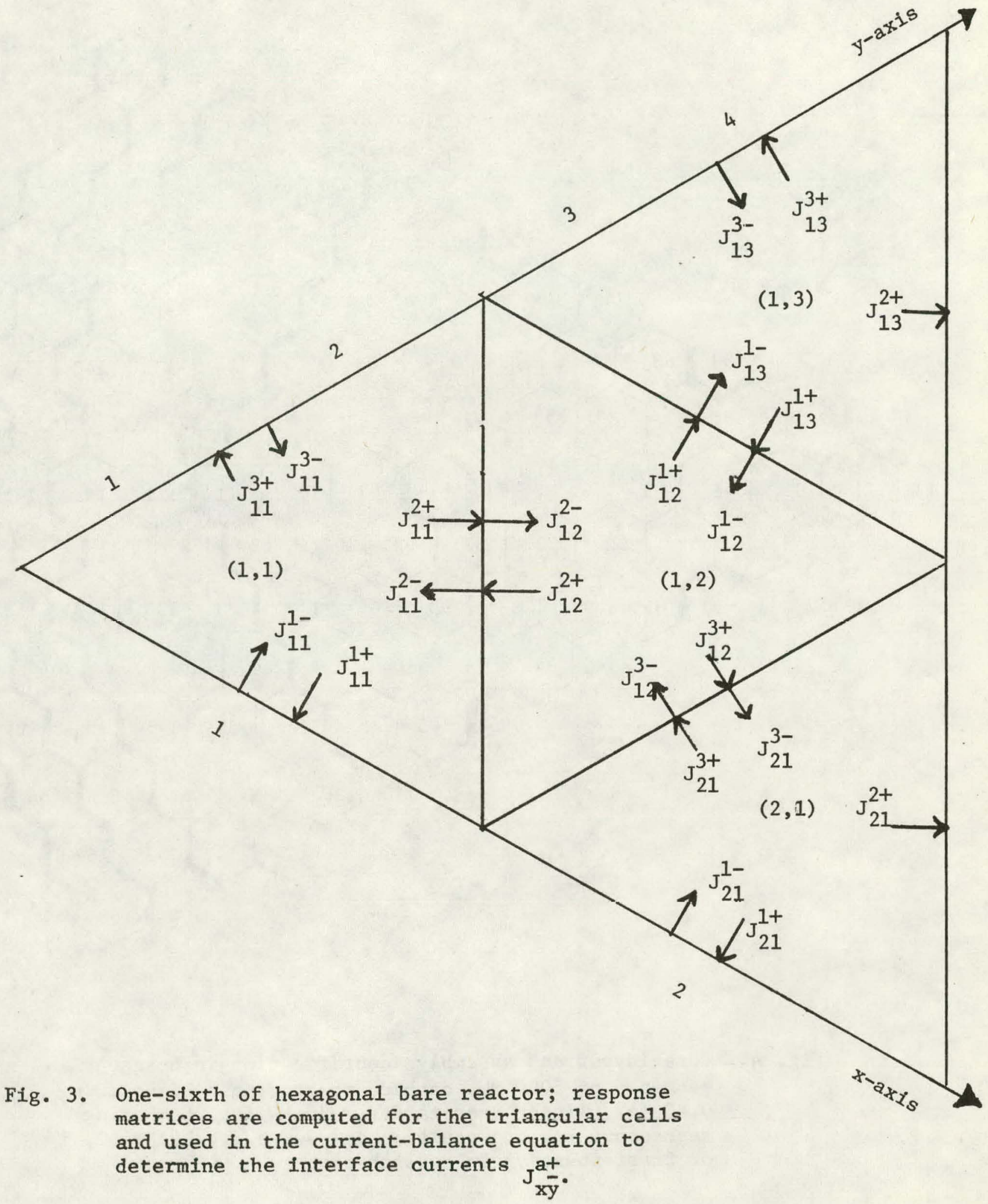




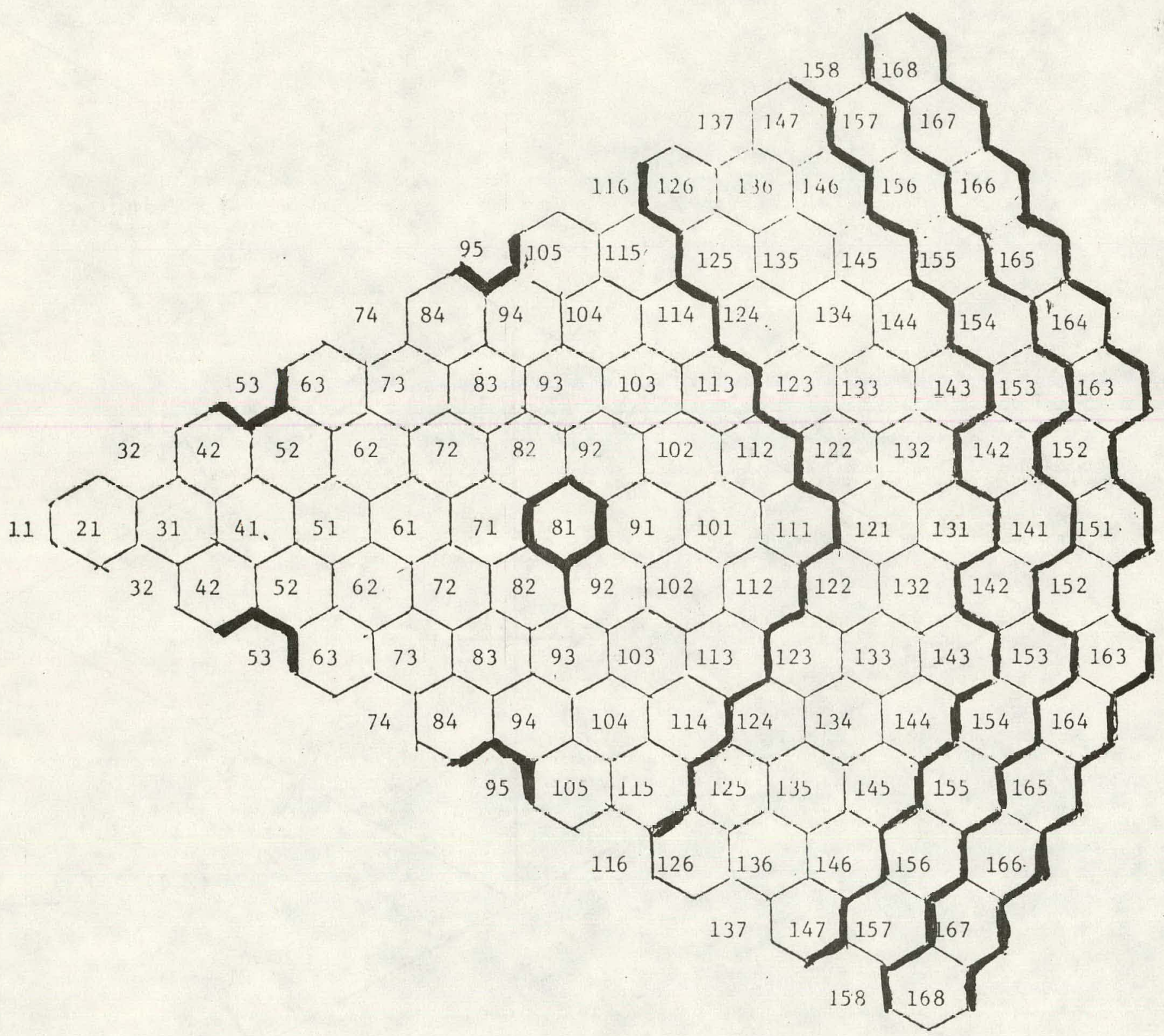

Fig. 4. Core layout and assembly identification in hexagonal geometry of 5000 MWt carbide reactor; pin diameter = $0.40 \mathrm{in}$., Iinear heat rating $=30 \mathrm{~kW} / \mathrm{ft}$. Numbering represents row and position in row, e.g., $121 \cong 12,1$ or first position in twelfth row. 


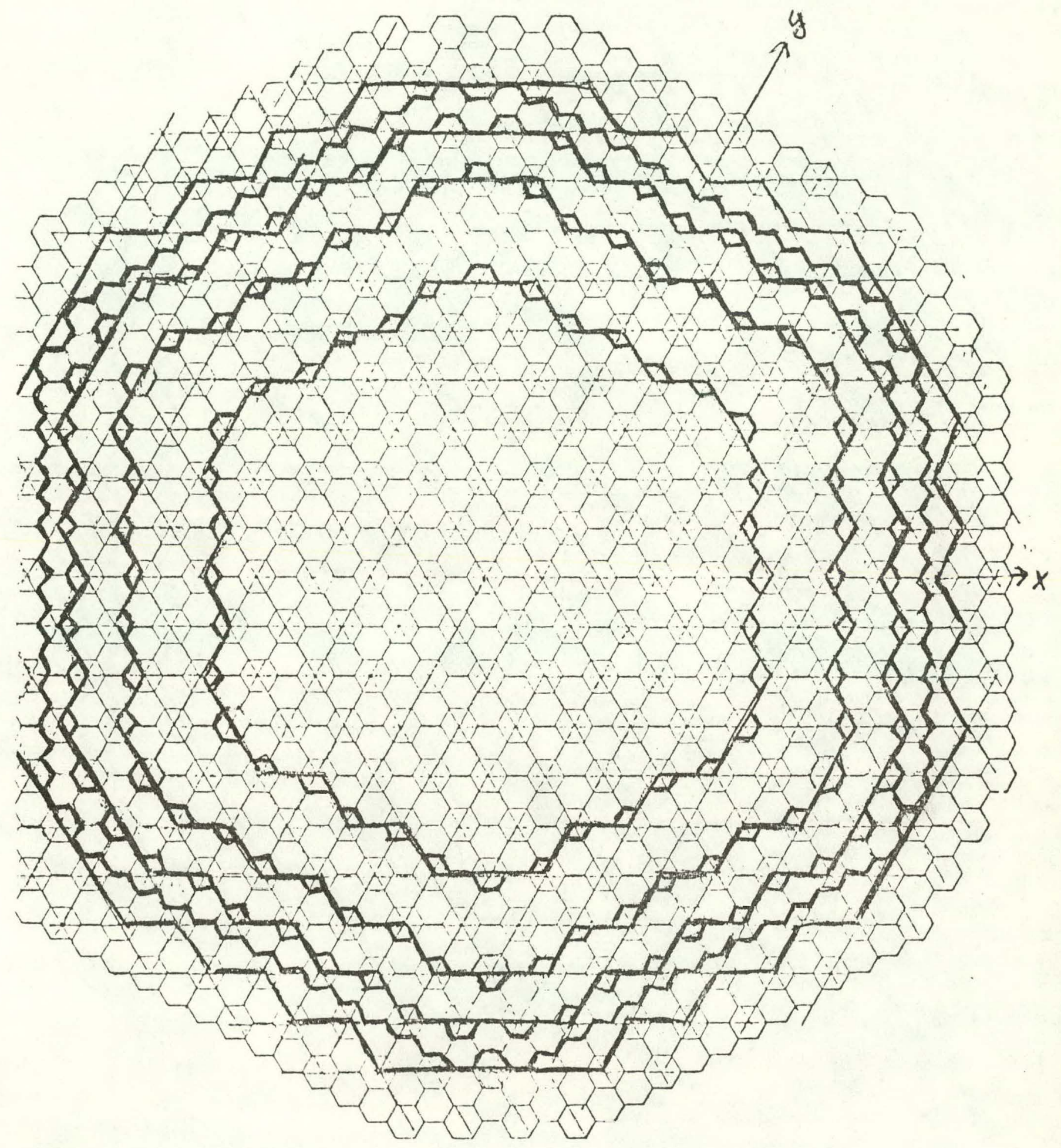

Fig. 5. A triangular cell decumposition of large hexagona1 model reactor. 


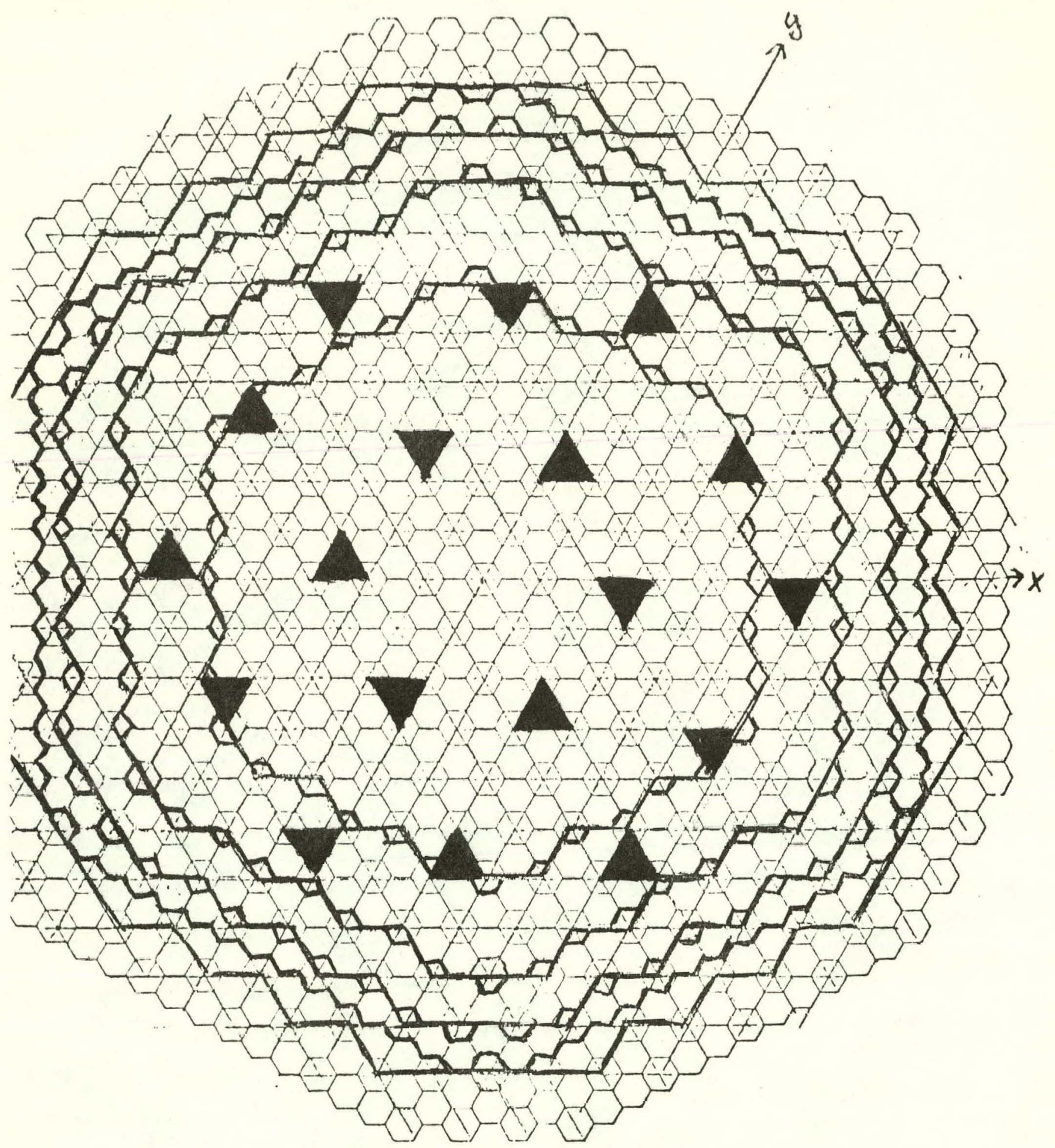

Fig. 6. Example of triangular cell placement of control poison in model with triangular geometry. 
Fig. 7. Schematic Diagram for Enrichment Search in Finite-Difference

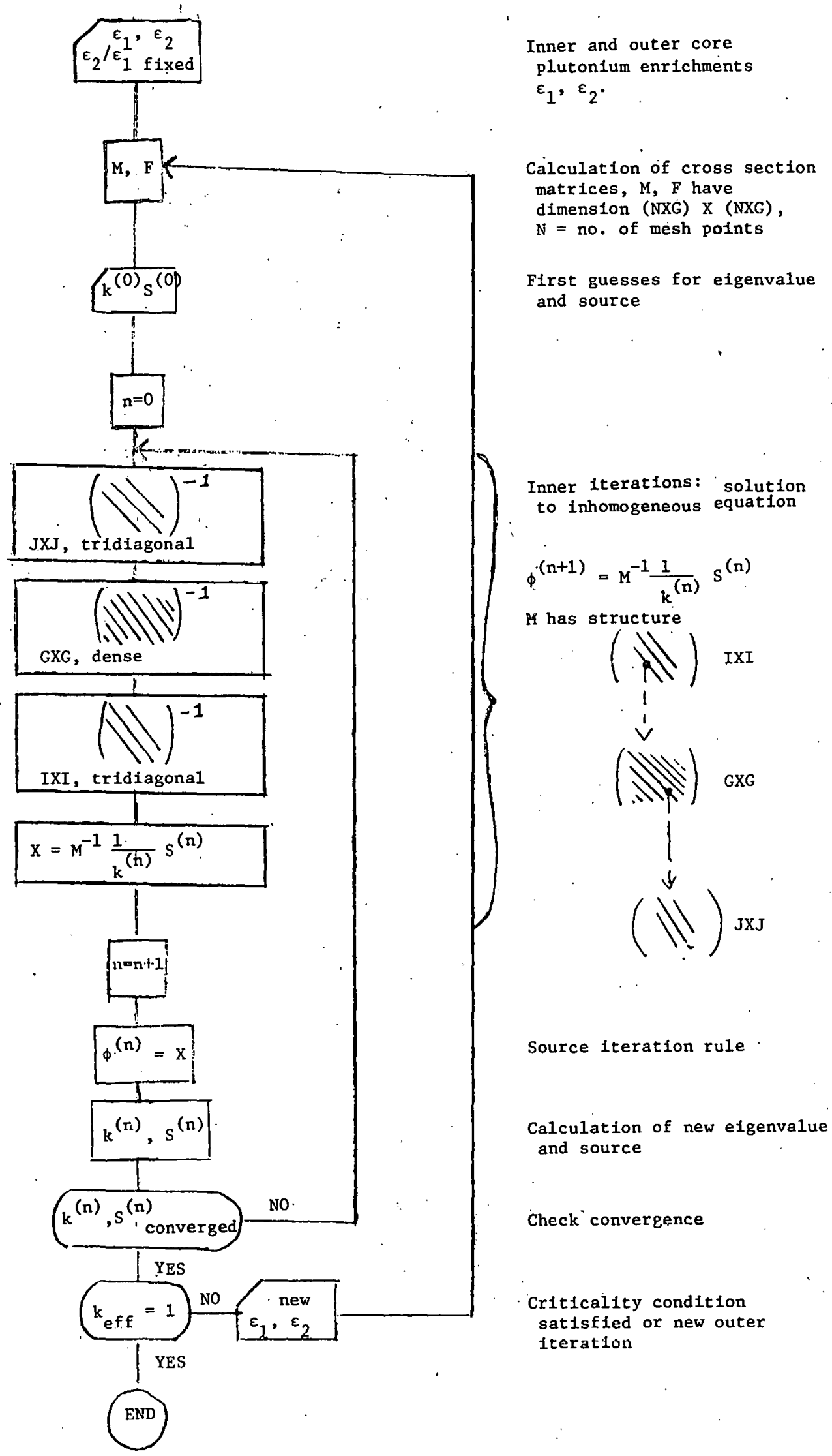


Fig. 8. Schemat1c Dlagram for Enrichment Search In Response Matrix Method *

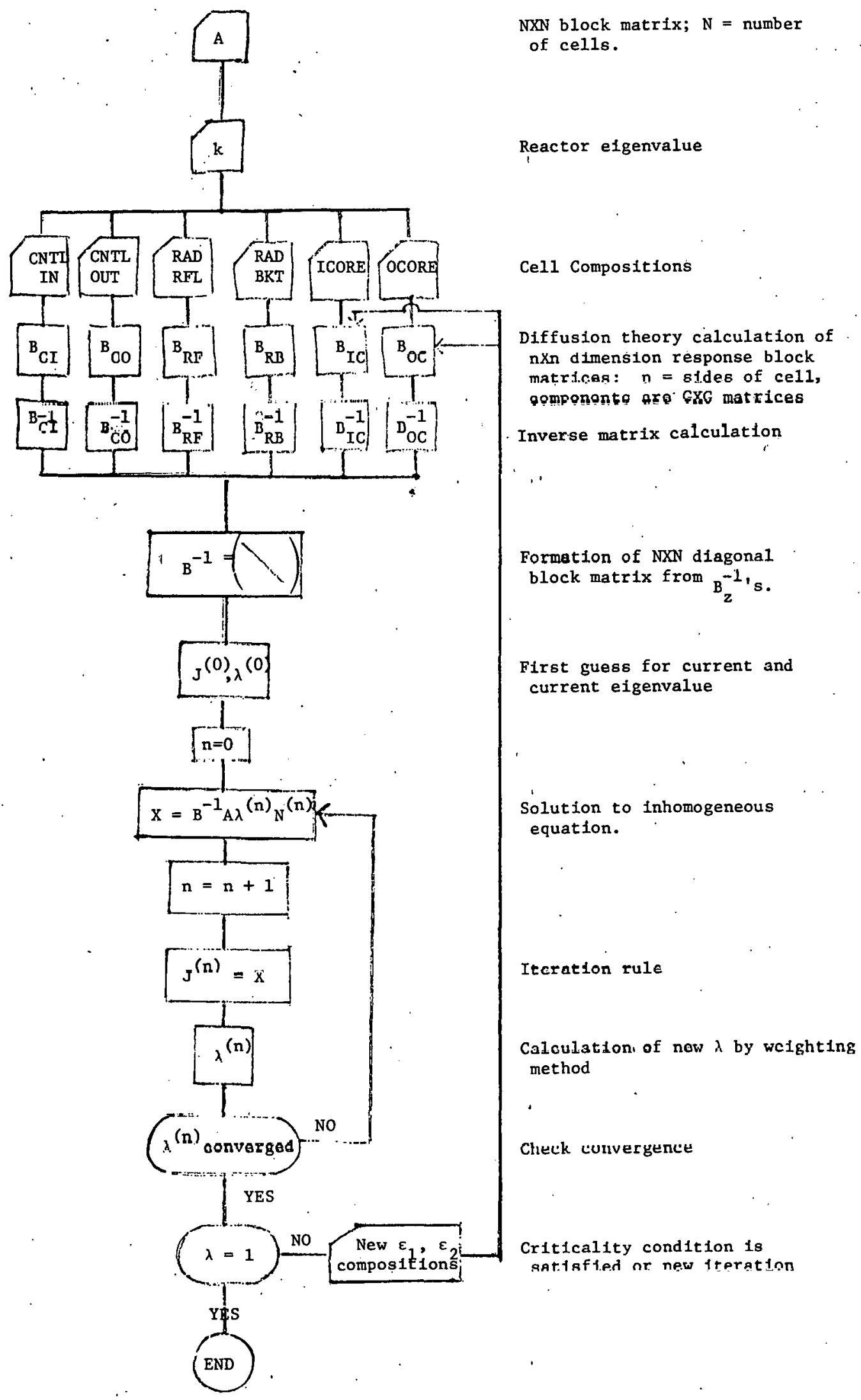




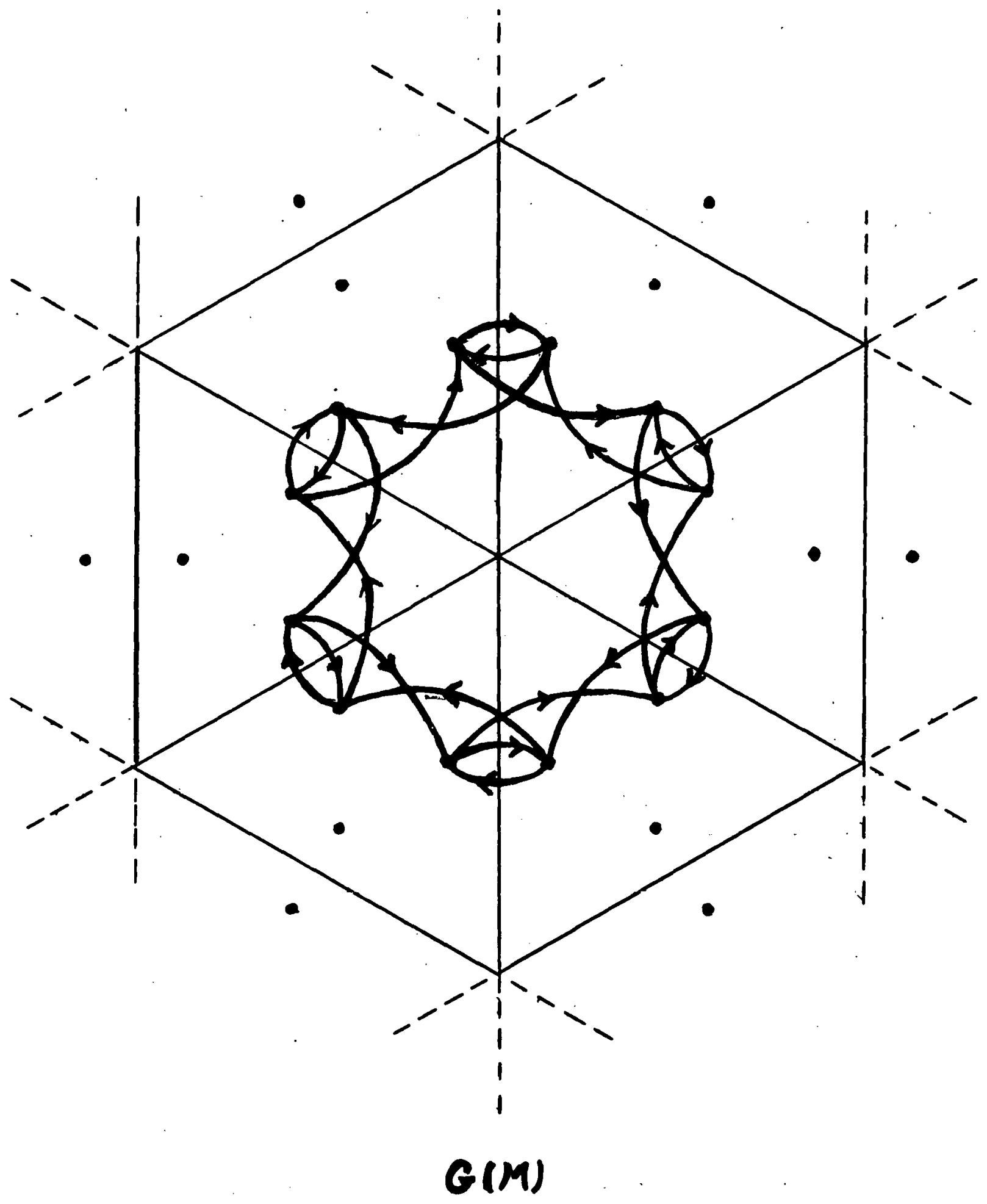

Fig. 9. A Portion of the Directed Graph G(M) for a

Two-Dimensional Model Based on Trtangular Cells. 


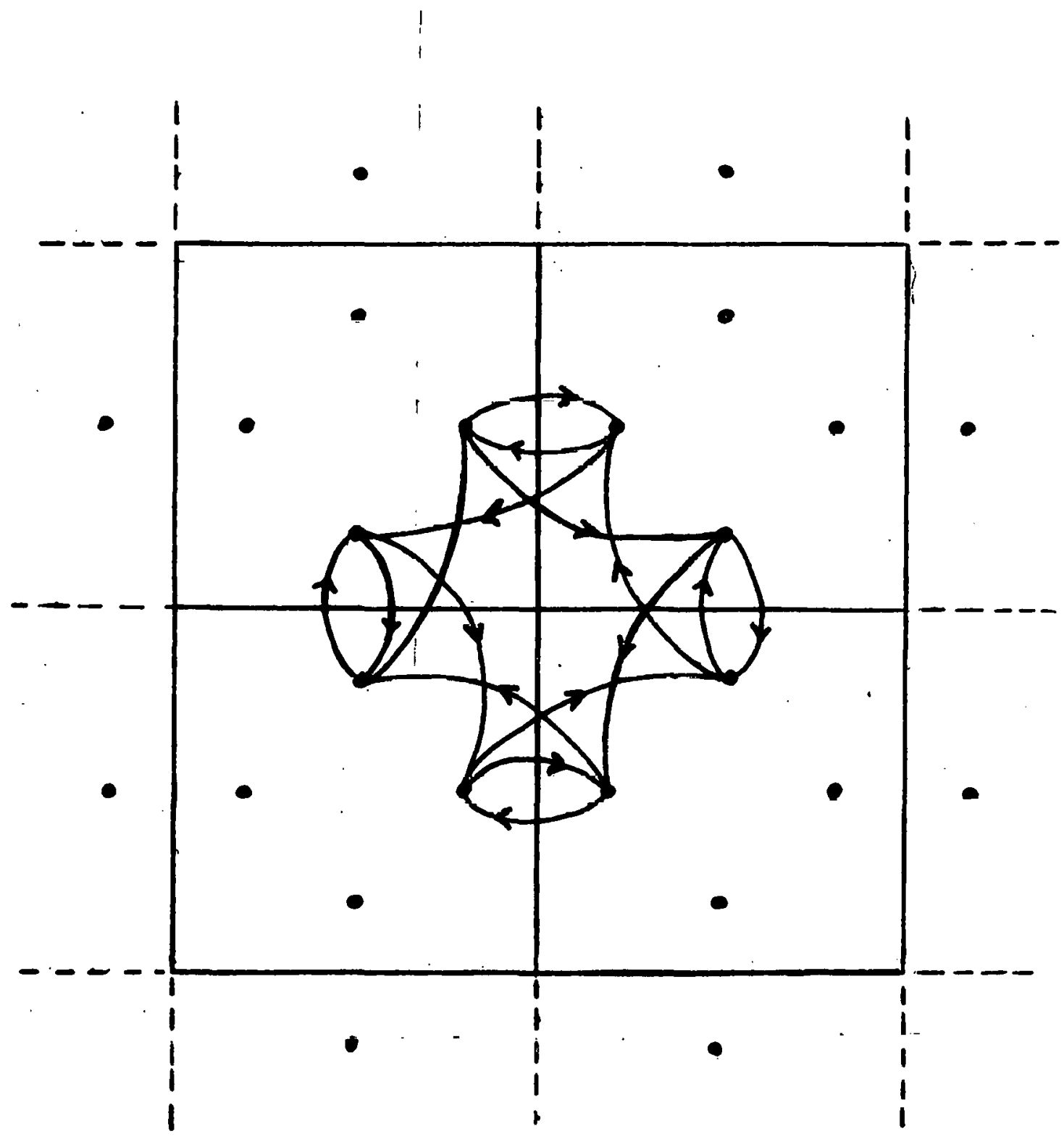

$G(M)$

Fig. 10. A-Portion of the Directed Graph $G(M)$ for a Two-Dimensional Model Based on Square Cells. 


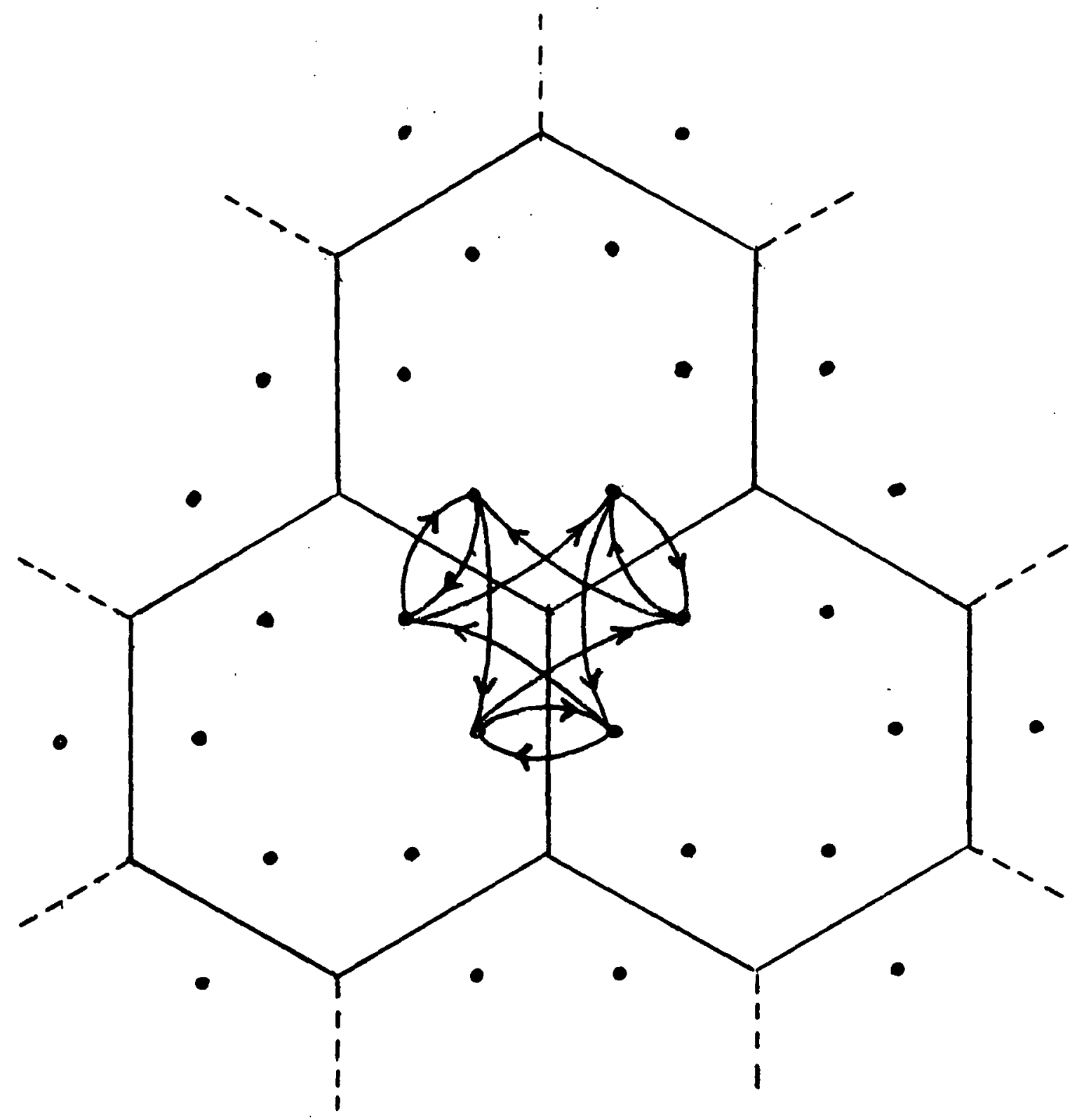

$G(M)$

Fig. 11. A Portion of the Directed Graph G(M) for a Two-Dimensional Model Based on Hexagonal Cells. 